\title{
A phase-field model for fracture of unidirectional fiber-reinforced polymer matrix composites
}

\author{
Funda Aksu Denli ${ }^{1}$ - Osman Gültekin ${ }^{1} \cdot$ Gerhard A. Holzapfel ${ }^{2,3} \cdot$ Hüsnü Dal ${ }^{1}$
}

Received: 29 August 2019 / Accepted: 22 December 2019 / Published online: 7 January 2020

(c) Springer-Verlag GmbH Germany, part of Springer Nature 2020

\begin{abstract}
This study presents a crack phase-field approach for anisotropic continua to model, in particular, fracture of fiber-reinforced matrix composites. Starting with the variational formulation of the multi-field problem of fracture in terms of the deformation and the crack phase fields, the governing equations feature the evolution of the anisotropic crack phase-field and the balance of linear momentum, presented for finite and small strains. A recently proposed energy-based anisotropic failure criterion is incorporated into the model with a constitutive threshold function regulating the crack initiation in regard to the matrix and the fibers in a superposed framework. Representative numerical examples are shown for the crack initiation and propagation in unidirectional fiber-reinforced polymer composites under Mode-I, Mode-II and mixed-mode bending. Model parameters are obtained by fitting to sets of experimental data. The associated finite element results are able to capture anisotropic crack initiation and growth in unidirectional fiber-reinforced composite laminates.
\end{abstract}

Keywords Fracture Failure $\cdot$ Fiber-reinforced polymers · FRP composites · Crack phase-field model · Anisotropic failure criterion

\section{Introduction}

Since the introduction of composite materials in the 1960's such as glass-polyester and carbon-epoxy, great success has been achieved in estimating the effective micromechanical properties of composites, the respective homogenized response and the plate theories for laminates. However, theories pertaining to the fracture of composite materials are not on par with the afore-mentioned theories in terms of their applicability and accuracy. Although a lot of efforts have been made over five decades, the prediction of composite failure still remains largely unsolved with plenty of uncertainties, as reviewed by Talreja [46].

Hüsnü Dal

dal@metu.edu.tr

1 Department of Mechanical Engineering, Middle East Technical University, Dumlupinar Bulvari 1, 06800 Çankaya, Ankara, Turkey

2 Institute of Biomechanics, Graz University of Technology, Stremayrgasse 16/II, 8010 Graz, Austria

3 Department of Structural Engineering, Norwegian University of Science and Technology (NTNU), 7491 Trondheim, Norway
Regarding fiber-reinforced polymers (FRPs), the failure mechanisms are, in general, related to (i) the mechanical behavior of the individual lamina and laminate as a whole and (ii) the direction of the loading. Compared with steel and other more conventional materials, the failure mechanism of FRP composites is much more complex and its prediction presents a tremendous challenge for engineers and researchers as they possess an inherent heterogeneity with distinct interfaces in their structure. The model approaches for the crack initiation and progression in composite materials can be divided into two categories: one is based on strength criteria (i.e. failure at a material point) and the other one is based on energy criteria (i.e. surface formation), see, e.g., Talreja and Singh [45].

According to strength-based criteria, micro-cracks form when the local stress (strain) state in a ply reaches a critical level. To date, several strength-based failure criteria that are rooted in metal fracture have been proposed for composite materials namely, Tsai and Hill [6,22], Tsai and Wu [47], Hashin [20] and Cuntze [13], just to mention a few. Expressed by quadratic polynomials, they involve strength values as material constants that need to be determined from experimental data. In principle, failure manifests when the elastic response in any combination of the stress components that 
exceed a threshold given by the respective criterion. Unlike the early maximum stress and strain criteria, the theories by Tsai-Hill, Tsai-Wu and Hashin take into account the possible stress interactions at failure. However, their use also leads to other issues. The Tsai-Hill criterion is based on the Hill criterion, which is basically an anisotropic extension of the von Mises [49] yield theory developed for isotropic materials such as metals. The predicted yield stress therein is the same in tension and compression, an appropriate conclusion for orthotropic metal sheets; however, such an assumption is far-fetched in regard to unidirectional composites as the mechanisms characterizing the failure under tension and compression are quite different from each other. In fact, a cluster of failed fibers in a cross-section is involved under tension and accompanied by some splits of fibers linking the neighboring cross-sections, whereas a local kink band facilitated by the micro-buckling deformation modes results in the failure of the composite under compressive loads, see, e.g., Argon [5]. Tsai-Wu account for unequal strengths in tension and compression that evokes the Bauschinger effect. This criterion assumes a scalar-valued function of stress components in regard to the failure surface characterized by an ellipsoid. The problem associated with this criterion is how to determine the inclination of the ellipsoid as the biaxial components of the strength tensor do not have a unique value, but hinge on the stress state. To overcome this difficulty, Hashin suggested many piece-wise smooth surfaces, each representing a distinct failure mode. In fact, the fiber failure in the criterion is decoupled from the matrix failure. Yet the problem of ascertaining the strength constants for compressive modes render the theory rather impractical. Later, Puck and Schürmann [37] proposed a more justifiable failure theory in the sense of model constants.

All of the afore-mentioned criteria regard the failure as a single event and consider composites as homogeneous solids. Besides, the failure plane is not explicitly influenced by the existence of fibers, i.e. the crack does not cut across the fibers. Nor do the distribution of fibers (uniform or nonuniform) and the nature of the matrix-fiber bond alter the critical tractions and the orientation of the crack surface. Furthermore, they are created on the basis of the traditional strength of the materials approach from the structural design aspect, and can only impart knowledge about the critical design points where the failure may occur. They fall short of describing differences in the crack initiation (as a material point failure process) and the crack propagation (as a surface growth process). As a consequence, the effect of ply thickness on the transverse cracking cannot be properly accounted by such criteria. Other problems are the impossibility of analytical characterization of local stress states except for a few cases and the conflict between the experimental data and the strength-based estimations, see Talreja and Singh [45].
Energy-based criteria originate from linear elastic fracture mechanics where the crack starts to grow when the energy release rate $G$ reaches a critical value $G_{\mathrm{c}}$ expressed by the equilibrium $G=G_{\mathrm{c}}$, as introduced by Griffith [16]. In a multiple cracking of a composite laminate, the progression of a crack located in between the plies is arrested at the interface and any further input of energy to the laminate leads to the formation of more ply cracks occurring elsewhere. In such a case, the conventional fracture mechanics approach requires modifications, e.g., the concept of finite fracture mechanics and variational stress analysis, which was among others presented by Hashin [21] and Nairn [32]. Aside from that, a strain energy-based failure criterion was suggested by Wolfe and Butalia [51] for a wide variety of unidirectional and symmetric laminates under biaxial loading.

The cohesive zone modeling (CZM) appears to be one of the most prevalent approaches used to mimic the mechanical failure of laminae which was presented by many including Turon et al. [48], Yang and Cox [52], Naghipour et al. [33] and Zhao et al. [54] for uni- and multi-directional composite laminates in Mode-I and Mode-II fracture. Likewise, there have been several successful applications of the extended finite element method (XFEM) by, e.g., Grogan et al. [17], Wang and Waisman [50] and Yazdani et al. [53] on the delamination of composite materials. Recent revelations by Dal et al. [14], Reinoso et al. [39], Alessi and Freddi [2] and Arterio et al. [4] highlight an alternative approach, namely the crack phase-field modeling to predict damage and failure of composite laminates. In contrast to CZM and XFEM, the crack phase-field approach utterly ignores the realization of discontinuities as the $2 \mathrm{D}$ crack surface smears out in a volume domain in 3D, which is determined by a specific field equation alongside the balance of linear momentum describing the elasticity of the solid. The well-known limitations of the classical fracture mechanics, e.g., curvilinear crack paths, crack kinking, branching angles, and multiple cracking are surmounted through a variational principal of the minimum energy, see Francfort and Marigo [15]. The thermodynamically consistent and algorithmically robust formulations of phase-field models were introduced in the seminal works by Miehe et al. [27,28]. The approach is modular and can be applied to non-standard solids exhibiting complex cracking mechanisms under multiphysics phenomena, see, e.g., Miehe et al. [29-31]. Ductile failure of elastoplastic materials is treated in Ambati et al. [3] and Borden et al. [8]. Anisotropic crack phase-field evolution has recently been considered by, e.g., $\mathrm{Li}$ et al. [25] and Teichtmeister et al. [44], which is based on the extended Cahn-Hilliard model, see Cahn and Hilliard [10], to account for the anisotropic surface energy emanating from the preferred directions in materials. Apart from that Clayton and Knap [11] and Nguyen et al. [36] proposed anisotropic phase-field models for polycrystals. The 


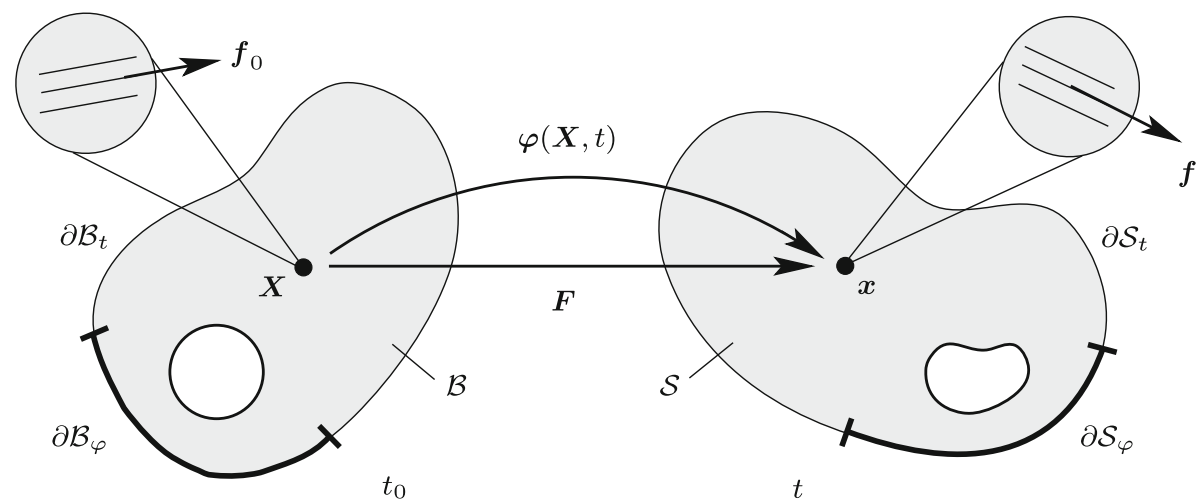

Fig. 1 Nonlinear deformation of a solid. The reference configuration $\mathcal{B} \in \mathbb{R}^{3}$ and the spatial configuration $\mathcal{S} \in \mathbb{R}^{3} ; \varphi: \mathcal{B} \times \mathcal{T} \mapsto \mathcal{S}$ is the nonlinear deformation map which maps the material point position $\boldsymbol{X} \in \mathcal{B}$ onto the spatial position $\boldsymbol{x}=\boldsymbol{\varphi}(\boldsymbol{X}, t) \in \mathcal{S}$, at time $t \in \mathbb{R}_{t}$. The deformation gradient $\boldsymbol{F}$ maps a Lagrangian line element $\mathrm{d} \boldsymbol{X}$ onto its

approach of Schreiber et al. [40] uses an anisoptropic geometric resistance to failure in the sense of Gültekin et al. [18].

For materials such as soft biological tissues and composite laminates, the anisotropic fracture is not only a geometrical phenomenon but also a mechanical event arisen from the fibrous content embedded in an otherwise isotropic matrix material, necessitating the use of an anisotropic crack driving force apart from directional geometric resistance. Hence, the current study follows the footsteps of the previous contributions by Gültekin et al. $[18,19]$ in which the anisotropic crack phase-field at finite strains was introduced. Incorporated was also a novel energy-based failure criterion based on the distinction of fibrous and matrix contributions to the elastic mechanical response. The current study, however, examines the fracture of the FRPs composed of a polymer matrix reinforced with fibers.

The article is organized as follows. Section 2 outlines the primary field variables with the corresponding finite strain kinematics and the diffusive features of the anisotropic crack phase-field. Section 3 is concerned with the variational formulation of the multi-field problem of fracture resulting in the coupled balance equations. Section 4 focuses on the anisotropic hyperelastic constitutive response reflecting the elastic mechanical behavior of the composite in the Eulerian framework. A brief account of the energy-based anisotropic failure criterion is also provided. In sect. 5, the representative numerical examples exhibit the capabilities of the model with regard to a standard problem of a transversely isotropic single edge-notched specimen under Mode-I and Mode-II loading scenarios and a realistic test case for a unidirectional laminate withstanding mixed-mode bending. Finally, Sect. 6 summarizes the article.

Eulerian counterpart $\mathrm{d} \boldsymbol{x}=\boldsymbol{F} \mathrm{d} \boldsymbol{X}$. The anisotropic micro-structure of the material point $\boldsymbol{X}$ is rendered by unidirectional fibers with the unit vector $f_{0}$. Likewise, the anisotropic micro-structure of the spatial point $\boldsymbol{x}$ is described by $\boldsymbol{f}$, as the spatial counterpart of $\boldsymbol{f}_{0}$

\section{Basics of the multi-field problem of fracture}

This section lays bare the primary field variables, namely the crack phase-field $d$ and the deformation map $\varphi$ governing the diffusive crack evolution and the balance of linear momentum in a coupled manner. The framework is provided for the finite and small-strain settings which cover both the mechanical and phase-field problems.

\subsection{The primary field variables}

Let us consider a continuum body at time $t_{0} \in \mathcal{T} \subset \mathbb{R}$, which we refer to as the reference configuration, as designated by $\mathcal{B} \subset \mathbb{R}^{3}$, with the material point $\boldsymbol{X} \in \mathcal{B}$. Similarly, the deformed body at current time $t \in \mathcal{T} \subset \mathbb{R}$, which we refer to as the spatial configuration, is denoted by $\mathcal{S} \subset \mathbb{R}^{3}$ with the spatial point $x \in \mathcal{S}$ mapped via the deformation field $\varphi$, see Fig. 1. Thus,

$\varphi_{t}(X): \begin{cases}\mathcal{B} \times \mathcal{T} & \rightarrow \mathcal{S}, \\ (X, t) & \mapsto x=\varphi(X, t) .\end{cases}$

Along with the deformation field given in (1), the basic geometric mapping for the crack phase-field $d$ is expressed by

$d: \begin{cases}\mathcal{B} \times \mathcal{T} & \rightarrow[0,1], \\ (\boldsymbol{X}, t) & \mapsto d(\boldsymbol{X}, t),\end{cases}$

which interpolates between the intact $(d=0)$ and the ruptured $(d=1)$ state of the material. 
Fig. 2 Multi-field problem: a mechanical problem of deformation along with Dirichlet and Neumann-type boundary conditions, that is $\varphi=\bar{\varphi}$ and $\boldsymbol{P} \cdot \boldsymbol{N}=\overline{\boldsymbol{T}}$, respectively; $\mathbf{b}$ evolution of the crack phase-field problem with the Neumann-type boundary condition $\mathcal{L} \nabla d \cdot N=0$

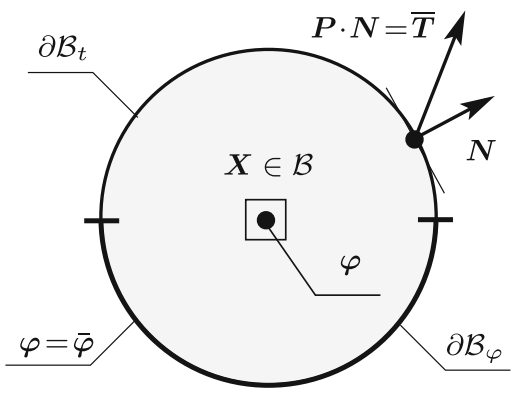

(a) Deformation field

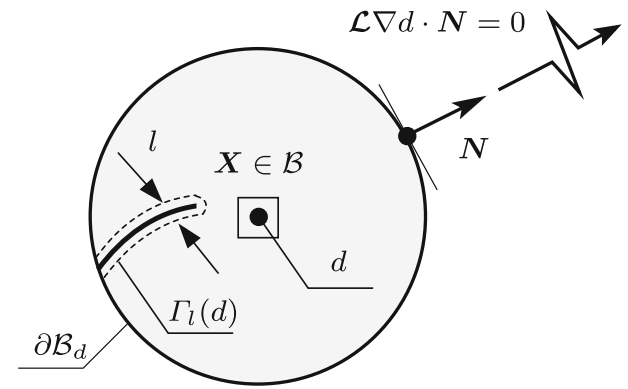

(b) Crack phase-field

\subsection{Kinematics of the mechanical problem}

We start with the description of the deformation gradient, i.e.

$$
\boldsymbol{F}=\nabla \boldsymbol{\varphi}_{t}(\boldsymbol{X})
$$

which maps the unit Lagrangian line element $\mathrm{d} \boldsymbol{X}$ onto its Eulerian counterpart $\mathrm{d} \boldsymbol{x}=\boldsymbol{F} \mathrm{d} \boldsymbol{X}$. The gradient operators $\nabla(\bullet)$ and $\nabla_{x}(\bullet)$ denote the gradient operator with respect to the reference $\boldsymbol{X}$ and the spatial $\boldsymbol{x}$ coordinates, respectively. The determinant of $\boldsymbol{F}$, i.e. the Jacobian $J:=\operatorname{det} \boldsymbol{F}>0$, characterizes the map of an infinitesimal reference volume element onto the associated spatial volume element. The right and left Cauchy-Green tensors read

$\boldsymbol{C}=\boldsymbol{F}^{T} \boldsymbol{g} \boldsymbol{F}, \quad \boldsymbol{b}=\boldsymbol{F} \boldsymbol{G}^{-1} \boldsymbol{F}^{T}$,

which measure the deformation in the Lagrangian and Eulerian configurations, respectively. Furthermore, we adopt the formalism in the sense of Marsden and Hughes [26] and equip the two manifolds, namely $\mathcal{B}$ and $\mathcal{S}$ with the covariant reference and spatial metric tensors $\boldsymbol{G}=\delta_{I J} \boldsymbol{E}^{I} \otimes \boldsymbol{E}^{J}$ and $\boldsymbol{g}=\delta_{i j} \boldsymbol{e}^{i} \otimes \boldsymbol{e}^{j}$, respectively, where $\delta_{I J}$ and $\delta_{i j}$ are simply evaluated as the Kronecker deltas in the Cartesian coordinate system. The energy stored in a hyperelastic isotropic material is characterized by the three invariants

$I_{1}=\operatorname{tr} \boldsymbol{b}, \quad I_{2}=\frac{1}{2}\left[I_{1}^{2}-\operatorname{tr}\left(\boldsymbol{b}^{2}\right)\right], \quad I_{3}=\operatorname{det} \boldsymbol{b}$.

The anisotropic response of a unidirectional FRP composite requires the description of an additional invariant. To this end, we introduce a reference unit vector $f_{0}$ for the fiber orientation in the reference configuration and its spatial counterpart

$$
f=\boldsymbol{F} \boldsymbol{f}_{0},
$$

which idealizes the micro-structure of the unidirectional FRP composite. We can express the related Lagrangian and Eulerian forms of the structure tensors $\boldsymbol{A}$ and $\boldsymbol{A}_{f}$ as follows
$\boldsymbol{A}=\boldsymbol{f}_{0} \otimes \boldsymbol{f}_{0}$ and $\boldsymbol{A}_{\boldsymbol{f}}=\boldsymbol{f} \otimes \boldsymbol{f}$.

The physically meaningful additional fourth-invariant

$I_{4}=f \cdot g f$,

measures the square of the stretch along the mean fiber direction in the unidirectional FRP composite.

\subsection{Kinematics of the phase-field problem}

The deformable domain for the concerning problem is associated with the deformation field as given in Fig. 2a. For a non-deformable domain, the gradient operator can simply be taken as $\nabla_{x}(\bullet)=\nabla_{X}(\bullet)=\nabla(\bullet)$. A sharp crack surface topology at a frozen time $t$ is defined by $\Gamma(d) \subset \mathbb{R}^{2}$ in the solid $\mathcal{B}$ through a surface integral $\Gamma(d)=\int_{\Gamma} \mathrm{d} A$. The hallmark of the crack phase-field approach is that it circumvents the cumbersome task of tracking such discontinuities and it approximates the surface integral by a volume integral, thereby creating a regularized crack surface $\Gamma_{l}(d)$, see also Fig. 2b such that

$$
\begin{aligned}
\Gamma_{l}(d) & =\int_{\mathcal{B}} \gamma(d, \nabla d) \mathrm{d} V \text { where } \\
\gamma(d, \nabla d) & =\frac{1}{2 l}\left(d^{2}+l^{2} \nabla d \cdot \nabla d\right),
\end{aligned}
$$

designates the isotropic crack surface density function, which satisfies the condition $\gamma(d, \boldsymbol{Q} \nabla d)=\gamma(d, \nabla d), \forall \boldsymbol{Q} \in \mathcal{O}(3)$. The tensor variable $\boldsymbol{Q}$ denotes the rotations in the orthogonal group $\mathcal{O}(3)$, containing rotations and reflections. The length-scale parameter $l$ controls the breadth of the crack. This approximation can be extended to a class of anisotropic materials as

$$
\begin{aligned}
& \Gamma_{l}(d)=\int_{\mathcal{B}} \gamma(d, \nabla d ; \mathcal{L}) \mathrm{d} V, \quad \text { where } \\
& \gamma(d, \nabla d ; \mathcal{L})=\frac{1}{2 l}\left(d^{2}+\nabla d \cdot \mathcal{L} \nabla d\right),
\end{aligned}
$$




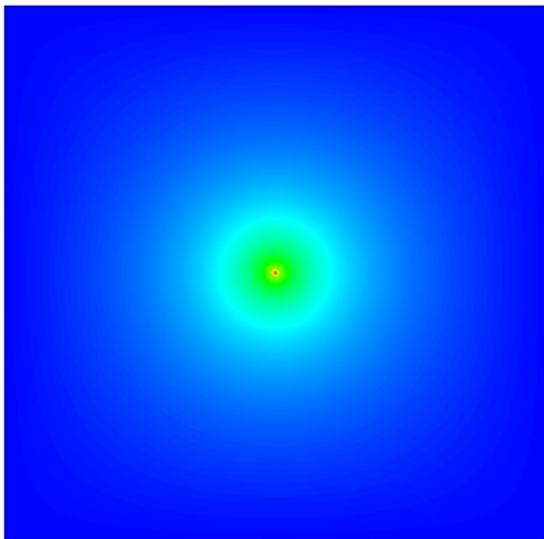

(a)

$$
\mathcal{L}=l^{2} \boldsymbol{I}
$$

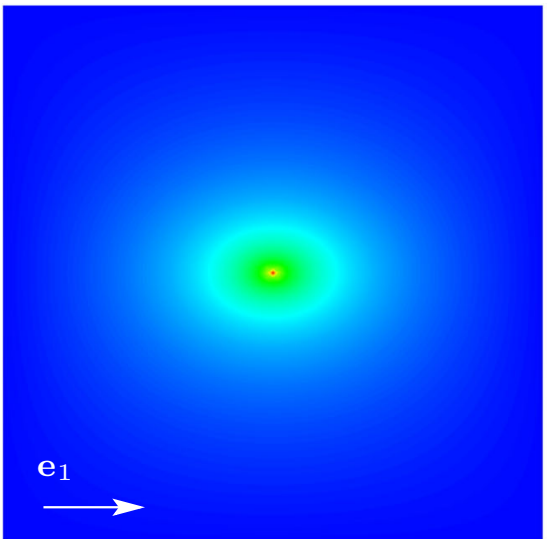

(b) $\quad \mathcal{L}=l^{2}\left(\boldsymbol{I}+\mathbf{e}_{1} \otimes \mathbf{e}_{1}\right)$

$d$

0

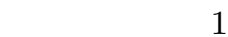

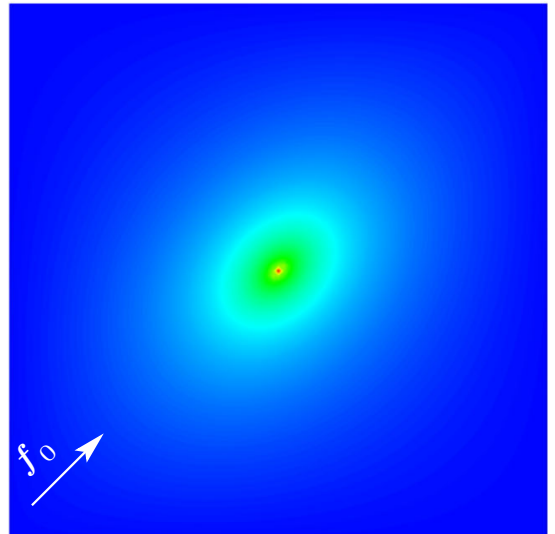

(c)

Fig. 3 Damage field on a square block: a isotropic damage field, $\mathbf{b}$ anisotropic damage field with fiber orientation $\theta=0^{\circ}$ stated by the unit base vector $\boldsymbol{e}_{1}, \mathbf{c}$ anisotropic damage field with fiber orientation $\theta=45^{\circ}$ given by the unit vector $\boldsymbol{f}_{0}$

is the anisotropic crack surface density function with the condition $\gamma(d, \boldsymbol{Q} \nabla d)=\gamma(d, \nabla d), \forall \boldsymbol{Q} \in \mathcal{G} \subset \mathcal{O}(3)$, where $\mathcal{G}$ designates a symmetry group as a subset of $\mathcal{O}(3)$. The second-order anisotropic structure tensor $\mathcal{L}$ is given as

$\mathcal{L}=l^{2}\left(\boldsymbol{I}+\omega_{\mathrm{f}_{0}} \boldsymbol{f}_{0} \otimes \boldsymbol{f}_{0}\right)$

which aligns the crack with the orientation of fibers in the continuum, see Fig. 3. Therein, the anisotropy parameter $\omega_{\mathrm{f}_{0}}$ regulates the transition from weak to strong anisotropy. For isotropic solids $\omega_{\mathrm{f}_{0}}=0$, whereas for a general anisotropic continuum, it must lie in an open range, i.e. $-1<\omega_{\mathrm{f}_{0}}<$ $\infty$ in order to satisfy the ellipticity condition for $\Gamma_{l}(d)$, see Gültekin et al. [19] for an elaborate discussion.

\subsection{Euler-Lagrange equations of the phase-field problem}

From a purely geometrical perspective, the boundary of the domain under interest can be decomposed into Dirichlet and Neumann-type boundaries such that $\partial \mathcal{B}=\partial \mathcal{B}^{d} \cup \partial \mathcal{B}^{q}$ and $\partial \mathcal{B}^{d} \cap \partial \mathcal{B}^{q}=\emptyset$. By considering (10), we can state the minimization principle as

$d(\boldsymbol{X})=\operatorname{Arg}\left\{\inf _{d \in \mathcal{W}} \Gamma_{l}(d)\right\}$,

along with the Dirichlet-type boundary constraint

$\mathcal{W}=\left\{d \mid d(\boldsymbol{X}) \in \mathcal{B} \wedge d=\hat{d}\right.$ on $\left.\partial \mathcal{B}^{d}\right\}$.

Whilst an already existing crack is given by $\hat{d}=1$, the intact state is described by $\hat{d}=0$. Although the boundary value problem admits any meta-states $\hat{d} \in[0,1]$ on $\partial \mathcal{B}^{d}$, we confine ourselves for the two ideal states. The Euler-Lagrange equations are obtained after employing the minimization principle. Thus,

$\frac{1}{l}[d-\operatorname{Div}(\mathcal{L} \nabla d)]=0$ in $\mathcal{B}$,

$\mathcal{L} \nabla d \cdot N=0$ on $\partial \mathcal{B}^{q}$,

where the divergence term interpolates $d$ between the intact and the ruptured state of the material. In (14) $2, N$ denotes the unit surface normal oriented outward in the reference configuration. Solving only the phase-field problem in the purely geometrical context, a 2-D square block with $\hat{d}=1$ at the single centroidal point and $\mathcal{L} \nabla d=0$ on the sides of the block leads to damage fields depicted in Fig. 3 for various structure tensors $\mathcal{L}$ associated with different fiber orientations.

In order to demonstrate the influence of $w_{\mathrm{f}_{0}}$, four representative cracks for two different anisotropy factors, namely $w_{\mathrm{f}_{0}}=0$ in Fig. $4 \mathrm{a}, \mathrm{c}$ and $w_{\mathrm{f}_{0}}=10$ in Fig. $4 \mathrm{~b}, \mathrm{~d}$ are depicted. The introduced centroidal cracks $(\hat{d}=1)$ are vertical in the first and horizontal in the second row. When the anisotropy parameter equals to zero, i.e. $w_{\mathrm{f}_{0}}=0$, the crack smears isotropically so that the geometric resistance to crack propagation is identical in all directions. However, $w_{\mathrm{f}_{0}}=10$ smears the crack considerably more in the transverse plane towards the fiber direction $\boldsymbol{e}_{1}$, see Fig. $4 \mathrm{~b}$, whereas the minimum smearing occurs around the cracks parallel to the fiber direction, see Fig. 4d, as observed in the second column. This means that cracks propagating across the fibers are penalized and the crack propagating along the fibers are favored. This is due to the fact that the energy threshold for the cracks propa- 
Fig. 4 Damage field on a square block with a single fiber family whose orientation is characterized by the unit base vector $\boldsymbol{e}_{1}: \mathbf{a}, \mathbf{b}$ for a vertical centroidal crack; $\mathbf{c}, \mathbf{d}$ for a centroidal horizontal crack where $w_{\mathrm{f}_{0}}=0$ in the first column and $w_{\mathrm{f}_{0}}=10$ in the second column
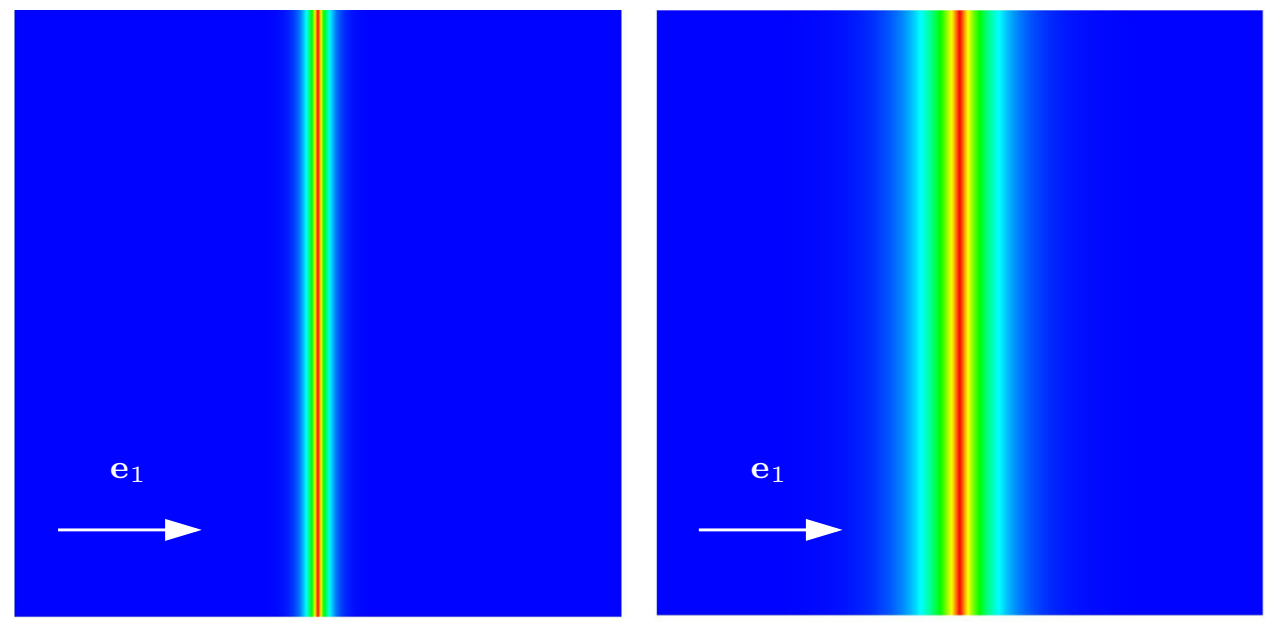

(a)

$w_{\mathrm{f}_{0}}=0$

(b)

$$
w_{\mathrm{f}_{0}}=10
$$

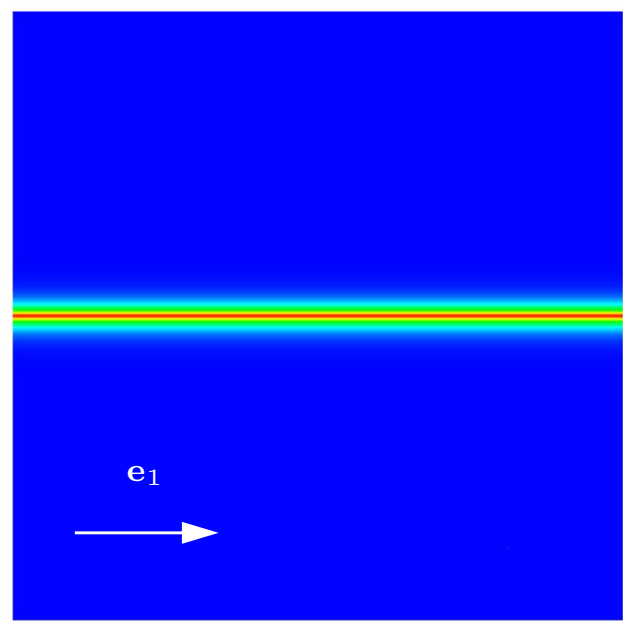

(d)

$$
w_{\mathrm{f}_{0}}=10
$$

(c)

$$
w_{\mathrm{f}_{0}}=0
$$

$d$ gating across the fibers are higher for $w_{f_{0}}>0$. The converse applies for $-1<w_{\mathrm{f}_{0}}<0$.

\section{Governing equations of the anisotropic fracture}

This section deals with the coupled equations of the elasticfracture problem for finite and small strains, where the classical balance of linear momentum is accompanied by the evolution equation of the crack phase-field; the strong forms of the boundary-value problem are presented.

\subsection{Rate-dependent variational formulation based on power balance}

\subsubsection{Finite-strain setting}

As a point of departure, we introduce the viscous rate-type potential $\Pi_{\eta}$ as

$\Pi_{\eta}=\mathcal{E}+\mathcal{D}_{\eta}-\mathcal{P}$

The first term $\mathcal{E}$ on the right-hand side of (15) represents the rate of energy storage functional, i.e.

$\mathcal{E}(\dot{\boldsymbol{\varphi}} ; \dot{d})=\int_{\mathcal{B}}(\boldsymbol{P}: \dot{\boldsymbol{F}}-f \dot{d}) \mathrm{d} V$, 
where the work conjugate variables to $\varphi$ and $d$ are the first Piola-Kirchhoff stress tensor $\boldsymbol{P}$ and the scalar energetic force $f$, respectively, i.e.

$\boldsymbol{P}=\partial_{\boldsymbol{F}} \Psi\left(\boldsymbol{g}, \boldsymbol{F}, \boldsymbol{A}_{\boldsymbol{f}} ; d\right), \quad f=-\partial_{d} \Psi\left(\boldsymbol{g}, \boldsymbol{F}, \boldsymbol{A}_{\boldsymbol{f}} ; d\right)$.

The free-energy function $\Psi$ defined in (17) characterizes a degrading continuum with

$\Psi\left(\boldsymbol{g}, \boldsymbol{F}, \boldsymbol{A}_{\boldsymbol{f}} ; d\right):=g(d) \Psi_{0}\left(\boldsymbol{g}, \boldsymbol{F}, \boldsymbol{A}_{\boldsymbol{f}}\right)$,

where $\Psi_{0}$ is the effective free-energy function of the hypothetically intact solid. In (18), a monotonically decreasing quadratic degradation function, i.e.

$g(d):=(1-d)^{2}$,

describes the degradation of the solid with the evolving crack phase-field parameter $d$ together with the following growth conditions:

$g^{\prime}(d) \leq 0 \quad$ with $\quad g(0)=1, g(1)=0, g^{\prime}(1)=0$.

The first condition ensures degradation, while the second and third condition set the limits for the intact and the ruptured state, and the final condition ensures the saturation at $d \rightarrow 1$. The second term $\mathcal{D}_{\eta}$ on the right-hand side of (15) is a viscous regularized dissipation functional due to fracture, i.e.

$\mathcal{D}_{\eta}(\dot{d}, \beta ; d)=\int_{\mathcal{B}}\left[\beta \dot{d}-\frac{1}{2 \eta}\langle\chi(\beta ; d, \nabla d)\rangle^{2}\right] \mathrm{d} V$,

where the artificial viscosity $\eta \geq 0$ regulates the scalar viscous over-stress $\chi$, which reads

$\chi(\beta ; d, \nabla d)=\beta-g_{\mathrm{c}}\left[\delta_{d} \gamma(d, \nabla d ; \mathcal{L})\right]$.

The Macaulay brackets in (21) filter out the positive values, $\chi>0$, while $g_{\mathrm{c}}$ in (22) stands for the critical fracture energy. Finally, the last term $\mathcal{P}$ on the right-hand side of (15) denotes the (classical) external power functional acting on the body according to

$\mathcal{P}(\dot{\boldsymbol{\varphi}})=\int_{\mathcal{B}} \rho_{0} \overline{\boldsymbol{\gamma}} \cdot \dot{\boldsymbol{\varphi}} \mathrm{d} V+\int_{\partial \mathcal{B}_{t}} \overline{\boldsymbol{T}} \cdot \dot{\boldsymbol{\varphi}} \mathrm{d} A$,

where $\rho_{0}, \bar{\gamma}$ and $\bar{T}$ represent the material density, the prescribed body force and the surface traction, respectively. Now, with the rate-type potential $\Pi_{\eta}$ at hand, we propose a mixed variational principle of the evolution problem as follows $\{\dot{\boldsymbol{\varphi}}, \dot{d}, \beta\}=\operatorname{Arg}\left\{\inf _{\dot{\varphi} \in \mathcal{W}_{\dot{\varphi}}} \inf _{\dot{d} \in \mathcal{W}_{\dot{d}}} \sup _{\beta \geq 0} \Pi_{\eta}\right\}$,

with the admissible domains for the primary variables

$\mathcal{W}_{\dot{\varphi}}=\left\{\dot{\boldsymbol{\varphi}} \mid \dot{\boldsymbol{\varphi}}=\mathbf{0} \quad\right.$ on $\left.\partial \mathcal{B}_{\varphi}\right\}$,

$\mathcal{W}_{\dot{d}}=\left\{\dot{d} \mid \dot{d}=0\right.$ on $\left.\partial \mathcal{B}_{d}\right\}$.

Afterwards, the variation of the potential $\Pi_{\eta}$ with respect to the fields $\{\dot{\boldsymbol{\varphi}}, \dot{d}, \beta\}$ along with simple algebraic manipulations via elimination and substitution of the respective terms (see Gültekin et al. [19] for more details) results in the strong form of the field equations, i.e.

$$
\begin{array}{ll}
\text { 1: } & \operatorname{Div} \boldsymbol{P}+\rho_{0} \overline{\boldsymbol{\gamma}}=\mathbf{0} \\
\text { 2: } & \eta \dot{d}=2(1-d) \overline{\mathcal{H}}-d+\operatorname{Div}(\mathcal{L} \nabla d) .
\end{array}
$$

The first equation in (26) simply describes the balance of linear momentum, whereas the latter states the evolution equation for the crack phase-field in which $\overline{\mathcal{H}}$ indicates the crack driving source term such that

$\overline{\mathcal{H}}=\frac{\Psi_{0}}{g_{\mathrm{c}} / l}$.

The evolution of the phase-field parameter can be recast into the form

$\dot{d}=\frac{1}{\eta}[2(1-d) \overline{\mathcal{H}}-d+\nabla d \cdot \mathcal{L} \nabla d]$,

\subsubsection{Small-strain setting}

The displacement field $\boldsymbol{u}=\boldsymbol{x}-\boldsymbol{X}$ is described at a material point $\boldsymbol{X} \in \mathcal{B} \subset \mathbb{R}^{3}$ and at time $t \in \mathcal{T}$, i.e.

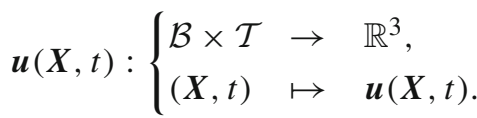

The rate of energy storage functional $\mathcal{E}$ on the right-hand side of (15) in the small-strain setting reads

$\mathcal{E}(\dot{\boldsymbol{u}} ; \dot{d})=\int_{\mathcal{B}}(\sigma: \dot{\boldsymbol{\varepsilon}}-f \dot{d}) \mathrm{d} V$,

where the stress tensor $\sigma$ is the work conjugate variable of the small-strain measure $\varepsilon=\operatorname{sym} \nabla \boldsymbol{u}$. In (30), the energetic force $f$ appears as the work conjugate of the damage variable $d$. The stress tensor $\sigma$ and the scalar energetic force $f$ are then expressed as

$\boldsymbol{\sigma}=\partial_{\varepsilon} \Psi(\varepsilon, A ; d), \quad f=-\partial_{d} \Psi(\varepsilon, A ; d)$. 
The term $f$ can also be interpreted as the local crack driving force. The free-energy function $\Psi$ in (31) characterizes a degrading continuum with

$\Psi(\boldsymbol{\varepsilon}, \boldsymbol{A} ; d):=g(d) \Psi_{0}(\boldsymbol{\varepsilon}, \boldsymbol{A})$,

where $\Psi_{0}$ is the effective free-energy function of the hypothetically intact solid. The regularized dissipation functional (21) and the scalar viscous over-stress function (22) remain unchanged. Finally, the (classical) external power functional $\mathcal{P}$ stated on the right-hand side of (15) can now be written in the form

$\mathcal{P}(\dot{\boldsymbol{u}})=\int_{\mathcal{B}} \rho_{0} \overline{\boldsymbol{\gamma}} \cdot \dot{\boldsymbol{u}} \mathrm{d} V+\int_{\partial \mathcal{B}_{t}} \overline{\boldsymbol{T}} \cdot \dot{\boldsymbol{u}} \mathrm{d} A$,

where $\rho_{0}, \bar{\gamma}$ and $\bar{T}$ represent the material density, the prescribed body force and the surface traction, respectively. With the expressions for the rate-type potential $\Pi_{\eta}$ at hand, we propose a mixed variational principle of the evolution problem as follows

$\{\dot{\boldsymbol{u}}, \dot{d}, \beta\}=\operatorname{Arg}\left\{\inf _{\dot{\boldsymbol{u}} \in \mathcal{W}_{\dot{u}}} \inf _{\dot{d} \in \mathcal{W}_{\dot{d}}} \sup _{\beta \geq 0} \Pi_{\eta}\right\}$,

with the admissible domains for the primary variables

$\mathcal{W}_{\dot{u}}=\left\{\dot{\boldsymbol{u}} \mid \dot{\boldsymbol{u}}=\mathbf{0} \quad\right.$ on $\left.\partial \mathcal{B}_{u}\right\}$,

$\mathcal{W}_{\dot{d}}=\left\{\dot{d} \mid \dot{d}=0 \quad\right.$ on $\left.\partial \mathcal{B}_{d}\right\}$.

Afterwards, the variation of the potential $\Pi_{\eta}$ with respect to the fields $\{\dot{\boldsymbol{u}}, \dot{d}, \beta\}$ and substitution of the respective terms (see Miehe et al. [27] for more details) we obtain the strong form of the field equations, i.e.

1: $\quad \operatorname{Div} \boldsymbol{\sigma}+\rho_{0} \overline{\boldsymbol{\gamma}}=\mathbf{0}$,

2: $\quad \eta \dot{d}=2(1-d) \overline{\mathcal{H}}-d+\operatorname{Div}(\mathcal{L} \nabla d)$.

\subsection{A note on the weak formulation and numerical implementation}

On the numerical side, a canonical Galerkin-type finite element procedure renders the weak forms of the coupled balance equations given in (26). The nonlinearities due to the geometry and the constitutive law, as subsequently described, necessitates a linearization process employed on the weak forms. Afterwards, an identical temporal and spatial discretization scheme is employed for the deformation map and the crack phase-field. The field variables are appropriately discretized with isoparametric shape functions so as to transform the continuous integral equations of the nonlinear weighted-residuals and their linearizations to a set of coupled, discrete algebraic equations. Finally, this set of algebraic equations is solved by a one-pass operator-splitting algorithm in a Newton-type iterative solver that successively updates the history field described by the failure criterion, the crack phase-field and the deformation field. For a more elaborate numerical treatment of the respective problem, the readers are referred to, e.g., Gültekin et al. [18,19].

\section{Constitutive equations of the multi-field problem}

In this section, we elucidate: (i) the constitutive equations that capture the nonlinear anisotropic response of a unidirectional FRP composite and (ii) the related energy-based anisotropic failure criterion capturing the state of the material at which the cracking starts/propagates.

\subsection{The constitutive model for the unidirectional FRP composites}

The free-energy function of isotropic solids can be modeled through the three invariants $I_{1}, I_{2}, I_{3}$, which constitute the integrity basis of the deformation tensors $\boldsymbol{C}$ or $\boldsymbol{b}$, see e.g., Spencer [43]. For incompressible materials the two invariants $I_{1}$ and $I_{2}$ are enough to describe the isotropic deformation.

For transversely anisotropic solids, one can introduce the additional set of invariants $I_{4}$ and $I_{5}$ with the help of the structural tensors that satisfy the objectivity requirement under superimposed rigid body rotations, see e.g., Betten [7], Boehler [9] and Schröder and Neff [41]. In unidirectional fiber-reinforced materials, the stored energy can be obtained in terms of a free energy of the unreinforced base matrix with the arguments $I_{1}$ and $I_{3}$ augmented by a storage function that involves the fourth invariant $I_{4}$ related to the fiber stretch. The latter function is also known as the standard reinforcing model, see Qiu and Pence [38]. A similar approach can be adopted for the modeling of soft biological tissues, see, e.g., Holzapfel et al. [24].

To characterize the local anisotropic mechanical response of a unidirectional FRP composite, the free-energy function can be stated as

$\Psi_{0}\left(\boldsymbol{g}, \boldsymbol{F}, \boldsymbol{A}_{\boldsymbol{f}}\right):=\Psi_{0}^{\mathrm{iso}}\left(J, I_{1}\right)+\Psi_{0}^{\mathrm{ani}}\left(I_{4}\right)$.

For the isotropic part of the mechanical response of the compressible polymer matrix we adopt the generic compressible neo-Hookean free-energy function. Thus,

$\Psi_{0}^{\text {iso }}\left(J, I_{1}\right):=\frac{\lambda}{2}(\ln J)^{2}+\frac{\mu}{2}\left(I_{1}-2 \ln J-3\right)$. 
For the anisotropic part we use the standard reinforcing model in the sense of Qiu and Pence [38], i.e.

$\Psi_{0}^{\mathrm{ani}}\left(I_{4}\right):=\frac{\mu_{\mathrm{f}}}{4}\left(I_{4}-1\right)^{2}$.

In (38), $\lambda$ denotes the Lamé's first constant, whereas $\mu$ denotes the Lamé's second constant or the shear modulus. In the anisotropic term (39), $\mu_{\mathrm{f}}$ stands for a stress-like material parameter associated solely with the fibrous content.

Let us now exploit the Coleman-Noll procedure on the Clausius-Planck inequality, and use the form of the freeenergy function $\Psi$, as introduced in (18), so that we can retrieve the Kirchhoff stress tensor $\boldsymbol{\tau}$ as

$\boldsymbol{\tau}:=\boldsymbol{P} \boldsymbol{F}^{\mathrm{T}}=2 \partial_{g} \Psi=g(d) \boldsymbol{\tau}_{0}, \quad \boldsymbol{\tau}_{0}=2 \partial_{g} \Psi_{0}$.

Therein, $g(d)$ is a monotonically decreasing quadratic degradation function as provided in (19). For the relevant nonlinear continuum mechanics used see, e.g., Holzapfel [23]. By substituting (37) along with (38) and (39) into the definition $(40)_{2}$ we get the stress expression for the intact material, i.e.

$\boldsymbol{\tau}_{0}=\lambda \ln J \boldsymbol{g}^{-1}+\mu\left(\boldsymbol{b}-\boldsymbol{g}^{-1}\right)+2 \psi_{4} \boldsymbol{f} \otimes \boldsymbol{f}$,

where we have introduced the (deformation-dependent) constitutive function $\psi_{4}$ by

$\psi_{4}:=\partial_{I_{4}} \Psi_{0}=\frac{\mu_{\mathrm{f}}}{2}\left(I_{4}-1\right)$

The change in the Kirchhoff stress tensor is provided by the elasticity tensor here given in the spatial form as

$\mathbb{C}:=4 \partial_{\text {gg }}^{2} \Psi=g(d) \mathbb{C}_{0}, \quad \mathbb{C}_{0}=4 \partial_{\text {gg }}^{2} \Psi_{0}$,

for which the effective elasticity tensor $\mathbb{C}_{0}$ has the explicit expression

$\mathbb{C}_{0}=\lambda \boldsymbol{g}^{-1} \otimes \boldsymbol{g}^{-1}+2(\mu-\lambda \ln J) \mathbb{I}_{\boldsymbol{g}^{-1}}+4 \psi_{44} \mathbb{M}$,

where the symmetric fourth-order identity tensor $\mathbb{I}_{g^{-1}}$ has the index representation $\left(\mathbb{I}_{\boldsymbol{g}^{-1}}\right)^{i j k l}=\left(\delta^{i k} \delta^{j l}+\delta^{i l} \delta^{j k}\right) / 2$. In addition, the constitutive function $\psi_{44}$ reads

$\psi_{44}:=\partial_{I_{4}} \psi_{4}=\frac{\mu_{\mathrm{f}}}{2}$,

and the fourth-order structure tensor takes on the following form

$\mathbb{M}:=f \otimes f \otimes f \otimes f$.

\subsection{Linearization of the constitutive model: small-strain setting}

The linearized form of the free-energy function (37) can be represented as

$\Psi_{0}(\boldsymbol{\varepsilon}, \boldsymbol{A}):=\Psi_{0}^{\text {iso }}(\boldsymbol{\varepsilon})+\Psi_{0}^{\text {ani }}(\boldsymbol{\varepsilon}, \boldsymbol{A})$.

The isotropic and the anisotropic parts take on the simple quadratic forms

$$
\begin{aligned}
\Psi_{0}^{\text {iso }}(\boldsymbol{\varepsilon}) & :=\frac{\lambda}{2}(\operatorname{tr} \boldsymbol{\varepsilon})^{2}+\mu(\boldsymbol{\varepsilon}: \boldsymbol{\varepsilon}), \\
\Psi_{0}^{\mathrm{ani}}\left(I_{4}\right) & :=\mu_{\mathrm{f}}(\boldsymbol{\varepsilon}: \boldsymbol{A})^{2} .
\end{aligned}
$$

The linear stress tensor $\sigma_{o}:=\partial_{\varepsilon} \Psi_{0}$ of the intact solid can then be derived from (48), i.e.

$\boldsymbol{\sigma}_{0}=\lambda(\operatorname{tr} \boldsymbol{\varepsilon}) \mathbf{1}+2 \mu \boldsymbol{\varepsilon}+2 \mu_{\mathrm{f}}(\boldsymbol{\varepsilon}: \boldsymbol{A}) \boldsymbol{A}$.

The related elasticity moduli $\mathbb{C}_{0}=\partial_{\varepsilon} \sigma_{0}$ of the intact solid can be derived as

$\mathbb{C}_{0}=\lambda \mathbf{1} \otimes \mathbf{1}+2 \mu \mathbb{I}+2 \mu_{\mathrm{f}} \boldsymbol{A} \otimes \boldsymbol{A}$.

Therein, the fourth order symmetric identity tensor $\mathbb{I}$ has the following index representation $\left(\mathbb{I}^{i j k l}=\left(\delta^{i k} \delta^{j l}+\delta^{i l} \delta^{j k}\right) / 2\right.$. The proposed Ansatz is the simplest form of transverse isotropy with only one additional material parameter $\mu_{\mathrm{f}}$ to describe the axial reinforcement due to unidirectional fibers. This particular choice assumes identical shear response in the planes including the fibers and the transverse plane. It also excludes the coupling effect between the bulk response and the fiber reinforcement.

\subsection{Energy-based anisotropic failure criterion}

Following Gültekin et al. [18,19], we start with the assumption that two distinct failure processes govern the cracking of the ground matrix and the fibers, whereby the anisotropic structure tensor $\mathcal{L}$ in (11) is additively decomposed as

$$
\begin{aligned}
\mathcal{L} & =\mathcal{L}^{\text {iso }}+\mathcal{L}^{\text {ani }} \quad \text { with } \\
\mathcal{L}^{\text {iso }} & =l^{2} \boldsymbol{I}, \quad \text { and } \quad \mathcal{L}^{\text {ani }}=l^{2} \omega_{\mathrm{f}_{0}} \boldsymbol{f}_{0} \otimes \boldsymbol{f}_{0} .
\end{aligned}
$$

Next we introduce $g_{\mathrm{c}}^{\text {iso }}$ and $g_{\mathrm{c}}^{\text {ani }}$ corresponding to the critical fracture energies attributed to the ground-matrix (isotropic) and the fibrous content (anisotropic) of FRP, respectively, which homogenize the distinct mechanical resistance of the respective interactions against rupture. Hence, the crack driving source term (27) can be decomposed as

$\overline{\mathcal{H}}^{\text {iso }}=\frac{\Psi_{0}^{\text {iso }}}{g_{\text {c }}^{\text {iso }} / l}, \quad \overline{\mathcal{H}}^{\text {ani }}=\frac{\Psi_{0}^{\text {ani }}}{g_{\text {ci }}^{\text {ani }} / l}$. 
For the rate-independent case for which $\eta \rightarrow 0$, the expressions (51) and (52) engender distinct evolution equations of the crack phase-field in relation to the ground-matrix and the fibrous content, i.e.

$$
\begin{aligned}
& 2(1-d) \overline{\mathcal{H}}^{\text {iso }}=d-\operatorname{Div}\left(\mathcal{L}^{\text {iso }} \nabla d\right), \\
& 2(1-d) \overline{\mathcal{H}}^{\text {ani }}=d-\operatorname{Div}\left(\mathcal{L}^{\text {ani }} \nabla d\right) .
\end{aligned}
$$

What remains is to superpose the two distinct failure processes (53), which leads to the rate-independent evolution equation of the phase-field, i.e.

$$
(1-d) \mathcal{H}=d-\frac{1}{2} \operatorname{Div}(\mathcal{L} \nabla d),
$$

along with the specific form of the dimensionless crack driving source term

$$
\begin{aligned}
\mathcal{H}(t) & =\max _{s \in[0, t]}[\langle\overline{\mathcal{H}}(s)-1\rangle], \\
\overline{\mathcal{H}} & =\overline{\mathcal{H}}^{\text {iso }}+\overline{\mathcal{H}}^{\text {ani }}
\end{aligned}
$$

Relation (55) indicates an irreversible and positive crack driving source term such that the maximum positive value of $\overline{\mathcal{H}}(s)-1$ is tracked down for the entire deformation history $s \in[0, t]$. The Macaulay brackets filter out the positive values for $\overline{\mathcal{H}}(s)-1$ and keeps the solid intact until the failure surface is reached, which denotes the energetic criterion proposed by Gültekin et al. [18,19]. Finally, in view of $(54)$, we specify the rate-dependent case, i.e.

$$
\underbrace{\eta \dot{d}}_{\text {Crack evolution }}=\underbrace{(1-d) \mathcal{H}}_{\text {Driving force }}-\underbrace{\left[d-\frac{1}{2} \operatorname{Div}(\mathcal{L} \nabla d)\right]}_{\text {Geometric resistance }},
$$

where the evolution of the crack is characterized by the balance between the crack driving force and the geometric resistance to the crack, see Miehe et al. [29]. A closer examination of (56) shows that the geometric resistance is directional dependent. In Fig. 4, for instance, the energy threshold ratio of the crack in the direction $f_{0}$ to that in the transverse direction to $f_{0}$ is $G_{\|} / G_{\perp}=\mathcal{L}_{11} / \mathcal{L}_{22}=$ $\left(w_{\mathrm{f}_{0}}+1\right) / 1$ leading to an isotropic crack resistance for $w_{\mathrm{f}_{0}}=0$.

\section{Representative numerical examples}

In this section we demonstrate the utility of the proposed diffusive fracture model for FRP composites. The model is
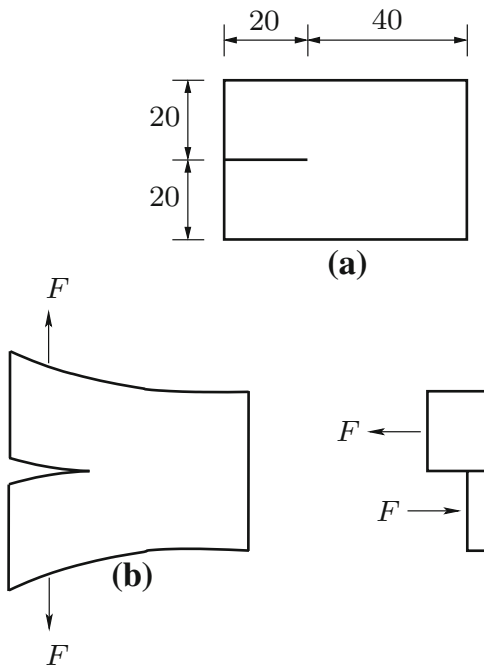

(a)

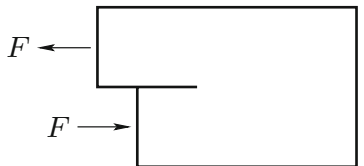

(c)

Fig. 5 Single edge-notched specimen with Mode-I and Mode-II loading: a dimensions of the specimen with a notch; $\mathbf{b}$ Mode-I load case; $\mathbf{c}$ Mode-II load case. All dimensions are in millimeter

capable of capturing anisotropic fracture, which is illustrated for a spectrum of benchmark problems such as single edgenotched specimens with various fiber orientations subjected to Mode-I and Mode-II loadings (Sect. 5.1). Although the strain levels remain small until the onset of cracking, the finite strain version of the theory is adopted in order to consider (i) the geometrical nonlinearities during the crack propagation phase, and (ii) the large strains observed around the crack tip. Finally a (more) realistic test case of a unidirectional laminate with an initial notch undergoing mixed-mode bending (MMB) is examined (Sect. 5.2).

\subsection{Mode-I and Mode-II tests for single edge-notched specimens with various fiber orientations}

Consider a rectangular plate with a horizontal notch placed in the middle of its height starting from the left edge. The dimensions of the specimen with the notch together with the Mode-I and Mode-II load cases are depicted in Fig. 5. For a discretization with 15000 standard displacement finite elements, an element size of $h=0.4 \mathrm{~mm}$ is used over the whole domain and the length-scale parameter $l$ is chosen to be 2.5 times the element size. For the analysis, the plane strain assumption is used, and only one element spans the thickness of the plate. The anisotropy parameter $\omega_{\mathrm{f}_{0}}$ is set to unity. The material is chosen to be a unidirectional AS4/3501-6 epoxy lamina with the (reference) fiber direction $\left[f_{0}\right]=[1,0,0]$ (horizontal fibers). In order to test how the proposed framework captures experimental data, we make use of a set of data provided by Soden et al. [42], and simulate the model response at a single Gauss point from which the model param- 
Table 1 Model parameters $\lambda, \mu, \mu_{\mathrm{f}}, g_{\mathrm{c}}^{\text {iso }}$ and $g_{\mathrm{c}}^{\text {ani }}$ with related values and units

\begin{tabular}{lll}
\hline Parameter & Value & Unit \\
\hline$\lambda$ & $5.2 \times 10^{3}$ & $(\mathrm{MPa})$ \\
$\mu$ & $4.04 \times 10^{3}$ & $(\mathrm{MPa})$ \\
$\mu_{\mathrm{f}}$ & $64.6 \times 10^{3}$ & $(\mathrm{MPa})$ \\
$g_{\mathrm{c}}^{\text {iso }}$ & 5.5 & $(\mathrm{MPamm})$ \\
$g_{\mathrm{c}}^{\text {ani }}$ & 80.0 & $(\mathrm{MPa} \mathrm{mm})$ \\
\hline
\end{tabular}

eters are obtained. For the related values and units see Table 1. Figure 6 compares the model results with the experimental data of the AS4/3501-6 epoxy lamina. The numerical results agree favorably with the experimental data both under tensile and compressive loads, a gradually diminishing mechanical response under compression is observed upon reaching the ultimate stress value.

The analyses for the Mode-I and Mode-II tests are now conducted for six different fiber angles namely $0^{\circ}, 15^{\circ}, 30^{\circ}$, $45^{\circ}, 60^{\circ}$ and $90^{\circ}$ (measured from the horizontal direction), which are shown in Fig. 7. In the Mode-I test, an incremental load is applied at the beginning for every specimen to find out the node-specific displacements, which are considered as the node-specific displacement increments during the rest of the analysis, thereby retaining the smoothness of the surfaces on which the displacements are exerted. As for Mode-II the computations are performed with constant displacement increments. The crack patterns pertaining to Mode-I and Mode-II are illustrated in Fig. 7, in which it can be seen that the propagation of the crack mostly follows the orientation of the fibers for all cases simulated.

The respective load-displacement curves are depicted in Fig. 8. The curves attributed to Mode-I (M1) suggest that a $0^{\circ}$ fiber angle exhibits the highest stiffness response, while a $90^{\circ}$ fiber angle experiences the highest strength. Part of the reason for such a distinct behavior may be that the flanks of the domain act, in a sense, as a cantilever beam under bending due to Mode-I, thereby leading to a higher stiffness value for the fiber orientation characterized by $0^{\circ}$ degree. As for a $90^{\circ}$ fiber angle, the branching of the crack upon the onset probably causes the highest strength among the cases tested. However, both the stiffness and strength values are in favor of the $0^{\circ}$ fiber angle when it comes to the Mode-II (M2) test. The crack needs to rupture more fibers on its way for a unit vertical distance.

\subsection{Mixed-mode bending of a unidirectional CFRP}

In this example the emphasis is on a thin rectangular carbon fiber-reinforced polymer composite (CFRP) with a notch in the middle subject to MMB. The information regarding the test apparatus, test procedure and the results are obtained from Crews and Reeder [12] and Naghipour et al. [35]. In MMB, the delamination of the CFRP occurs under combined influence of normal (Mode-I) and shear/sliding (Mode-II) stresses. MMB tests make it possible to describe the delamination resistance of a CFRP specimen and to account for the effects of combined stresses by using a single test apparatus. A geometrical sketch of the testing device consisting of a load $F$ and a loading lever with length $c$ is shown in Fig. 9a in the undeformed configuration.

A more detailed illustration of the loading acting on the hinge supports along with the superposition of the loads delineating Mode-I and Mode-II is shown in Fig. 10. Therein, $a$ stands for the initial crack length (distance between the loading direction and the crack tip) acting as a delamination initiator, whereas $L$ characterizes the specimen half-span. In particular, the loading position $c$ can be manipulated to generate a (pure) Mode-II loading case where $F$ is directly above the beam mid-span $(c=0)$. By removing the loading lever and pulling up the hinge, one can achieve a (pure) ModeI loading scenario. It is worth to note that the relationship between the deflection $\delta_{\mathrm{c}}$ at the specimen half-span, at the hinge $\delta_{\text {hinge }}$ and the total displacement $\delta_{\mathrm{MMB}}$ that occurs at the loading point is

$\delta_{\mathrm{MMB}}=\delta_{\mathrm{c}}+\frac{c}{L} \delta_{\text {hinge }}$ with $\delta_{\text {hinge }}=\delta_{\mathrm{c}}+\delta_{\text {Mode-I }}$,

where $\delta_{\text {Mode-I }}$ is the displacement at the hinge associated with Mode-I loading. For an illustration of the deformed state see Fig. 9b.

\subsubsection{Properties of the test specimen, experiments}

The used material for the test specimen is APC2-prepreg, which is composed of AS4-fibers (60\% of the total volume) embedded in a polyether ether ketone (PEEK) matrix. Each prepreg layer possesses a thickness of $140 \mu \mathrm{m}$. In total, 24 carbon/PEEK unidirectional laminae ([0] 24 ) are considered in the layup yielding a final specimen size of $25 \mathrm{~mm}$ width, $150 \mathrm{~mm}$ length and $3.12 \mathrm{~mm}$ thickness. Furthermore, a $50 \mathrm{~mm}$ film is placed as a delamination initiator in the midplane, as indicated in Fig. 11.

In accordance with the standards established by ASTM [1], Naghipour [34] conducted mixed-mode experiments by using the MMB testing device set up according to Fig. 9, and by applying a cross-head displacement rate of $0.5 \mathrm{~mm} / \mathrm{min}$. Therein, different mode mixtures were considered. The related lengths of the loading lever $c$ for each mode mixture, namely $30 \%$ and $50 \%$, are $98.5 \mathrm{~mm}$ and $65.0 \mathrm{~mm}$, respectively, and the resulting load-displacement $\left(F-\delta_{\mathrm{MMB}}\right)$ behavior of the unidirectional layup is shown as dashed curves in Fig. 12. 
Fig. 6 Numerical prediction versus experimental data for an AS4/3501-6 epoxy

lamina-stress $\sigma$ versus strain $\varepsilon$ in the 1 (horizontal) and 2 (vertical) direction: a tension in the 1 direction; $\mathbf{b}$ compression in the 2 direction

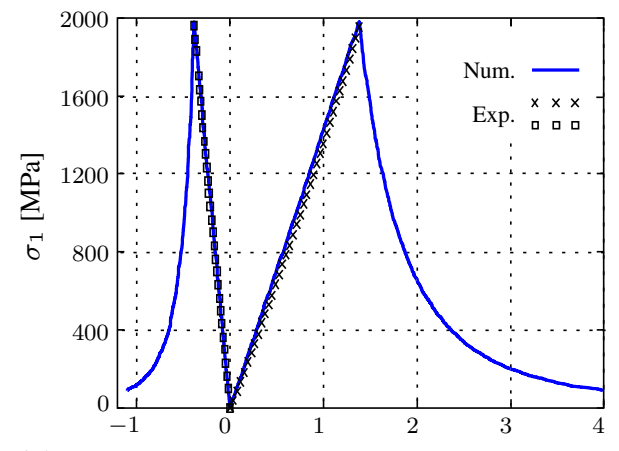

(a)

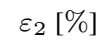

$\varepsilon_{1}[\%]$
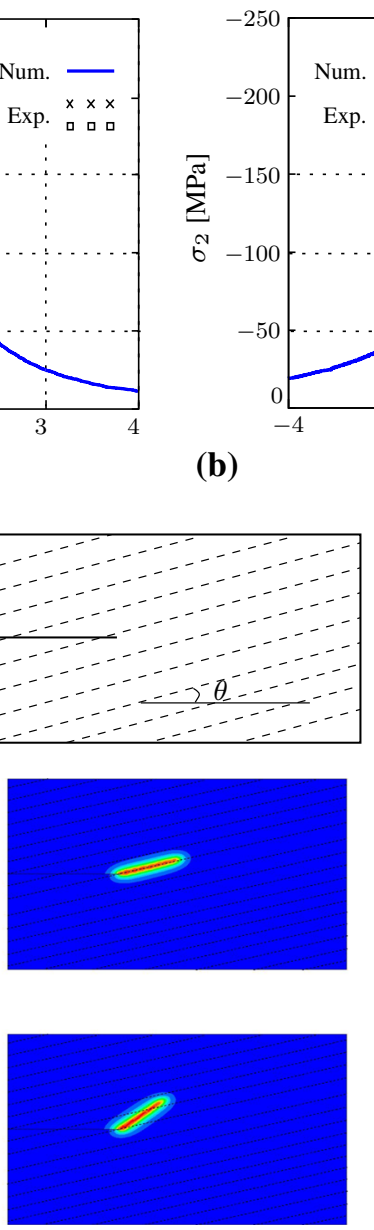

(b) $\theta=15^{\circ}$
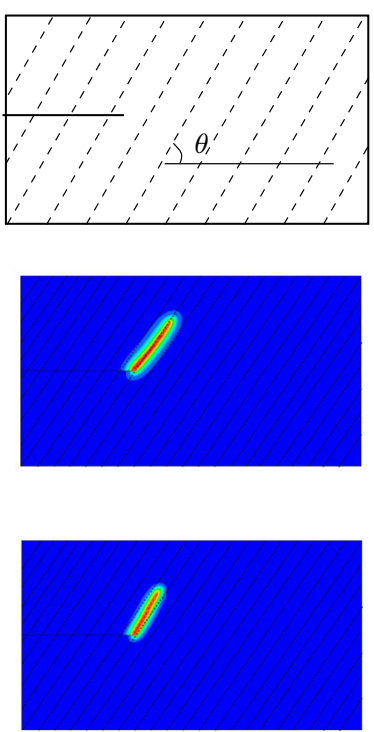

(e) $\theta=60^{\circ}$

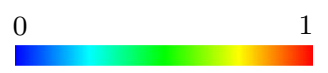

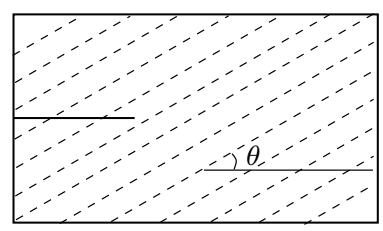
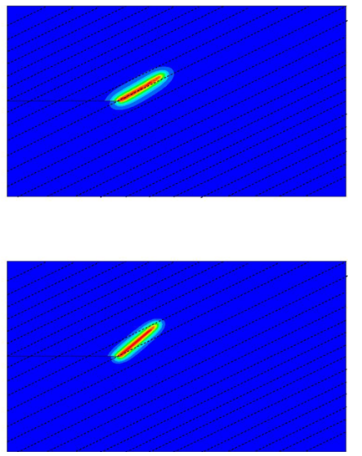

(c) $\quad \theta=30^{\circ}$
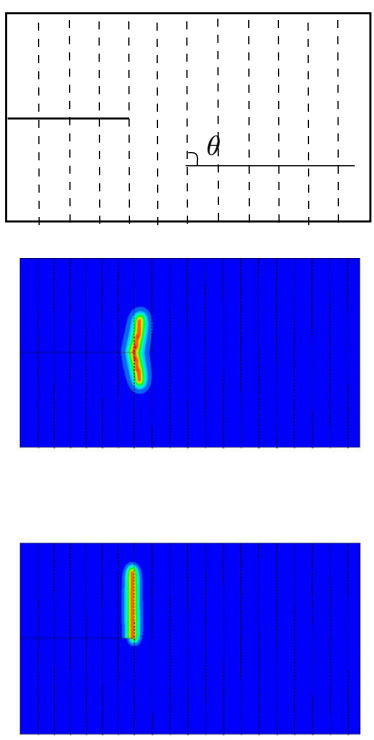

(f) 
Fig. 8 Relationships between load $F$ and displacement $u$ for Mode-I (M1) and Mode-II (M2) tests on single edge-notched specimens with various fiber angles, namely $\theta=0^{\circ}, 15^{\circ}$, $30^{\circ}, 45^{\circ}, 60^{\circ}$ and $90^{\circ}: \mathbf{a}, \mathbf{b}$ Mode-I; c, d Mode-II
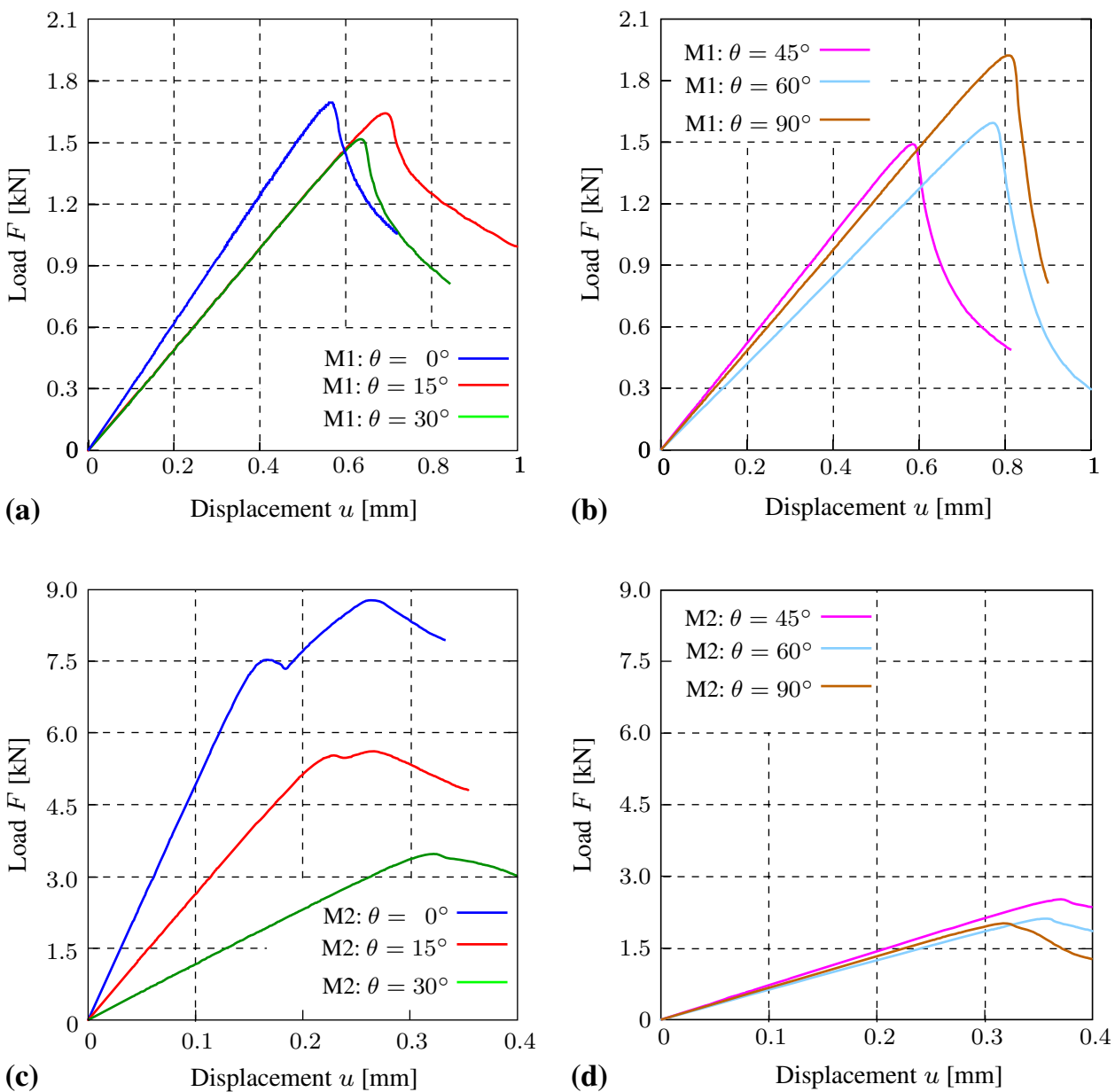

\subsubsection{Numerical simulation}

The material properties of the APC2 lamina are obtained at a Gauss point, which are summarized in Table 2, while the (reference) fiber direction is $\left[\boldsymbol{f}_{0}\right]=[1,0,0]$ (horizontal fibers). Figure 13 provides the fitting to the experimental data, i.e. the in-plane stress values pertaining to the fiber (horizontal) and transverse (vertical) directions, see Naghipour [34].

In accordance with the loading descriptions characterized in Fig. 9, an in silico replica of the specimen is made and then discretized. In order to better resolve the crack pattern the mesh is refined around the crack tip yielding a discretization of 6000 brick elements with an effective element size of $h=0.065 \mathrm{~mm}$ in the refined zone. Appropriate boundary conditions are applied to avoid rigid body motions, see Fig. 11. In the analyses the plane strain assumption is applied with a single element used in the direction of the width. Two sets of analyses are carried out: (i) with the anisotropy parameter $\omega_{\mathrm{f}_{0}}=1$ and (ii) with $\omega_{\mathrm{f}_{0}}=30$. Two different mode mixtures are taken into account in which the lengthscale $l$ is considered to be $0.15 \mathrm{~mm}$ satisfying the empirical requirement that $l \geq 2 h$. In other words, the length-scale parameter considered is greater than two times the minimum element size. The applied load $F$ and the chosen specimen half-span $L$ are $1 \mathrm{~N}$ and $62.5 \mathrm{~mm}$, respectively, from which the proportional loads exerted on the specimen are calculated. Afterwards, the displacement ratios corresponding to the calculated proportional loads are ascertained, and applied at the loading points, as depicted in Fig. 10. The lengths $c$, the proportional (hinge/middle) loads and the respective displacement ratios for each mode mixture are listed in Table 3. As a matter of fact, the analyses are carried out displacement-driven. The loading speed is $5 \mathrm{~mm} / \mathrm{min}$ with the time increment $\Delta t=0.5$ that runs until the onset of the macro-crack. From this point onward, the time increment is reduced to $\Delta t=0.05$, which is followed by $\Delta t=0.005$ during the crack propagation.

To obtain the corresponding load-displacement $\left(F-\delta_{\mathrm{MMB}}\right)$ curve from the points on which the loads are applied, the values of the displacements at the hinge $\delta_{\text {hinge }}$ and the middle $\delta_{\mathrm{c}}$ of the specimen together with the related reaction forces are stored during each analysis. The applied load $F$ is then calculated via the balance of moment with respect to the mid-point of the lever, i.e. $F=F_{\text {hinge }} L / c$, for every time 


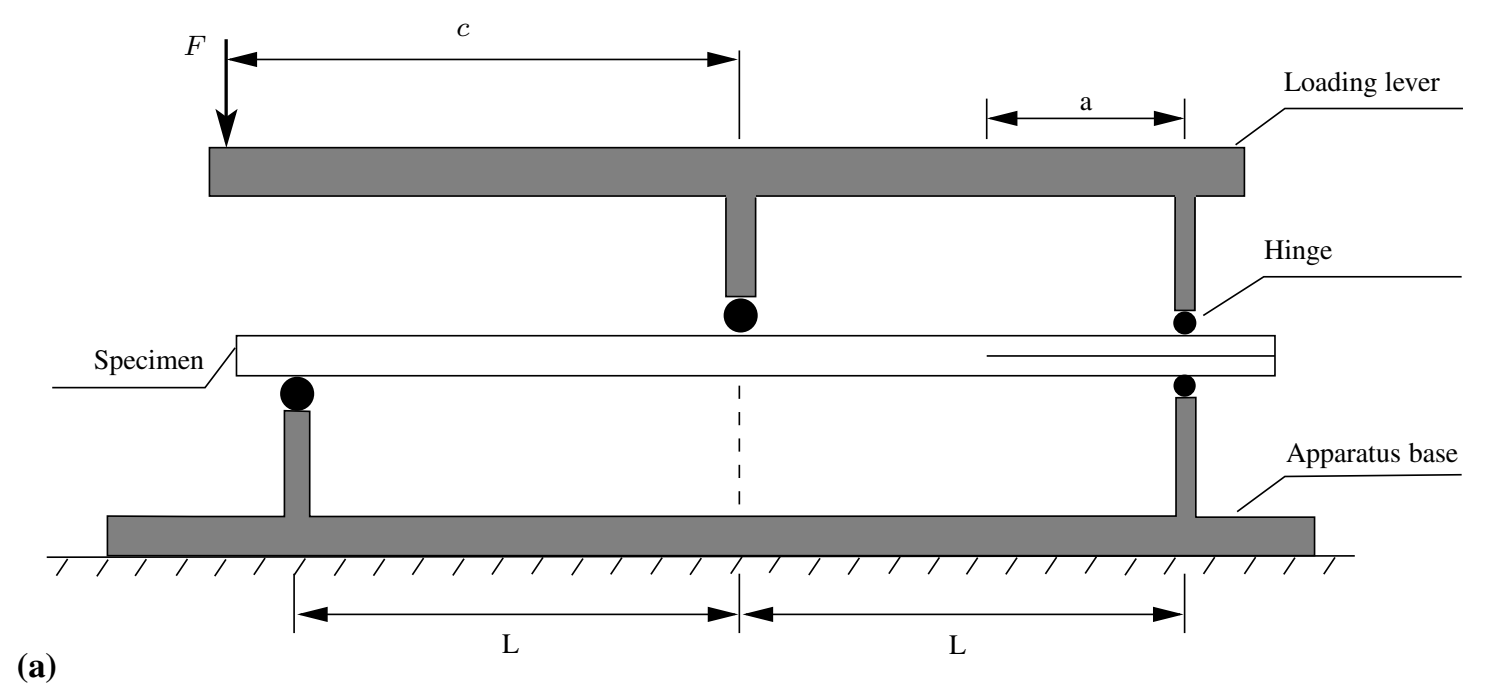

(a)

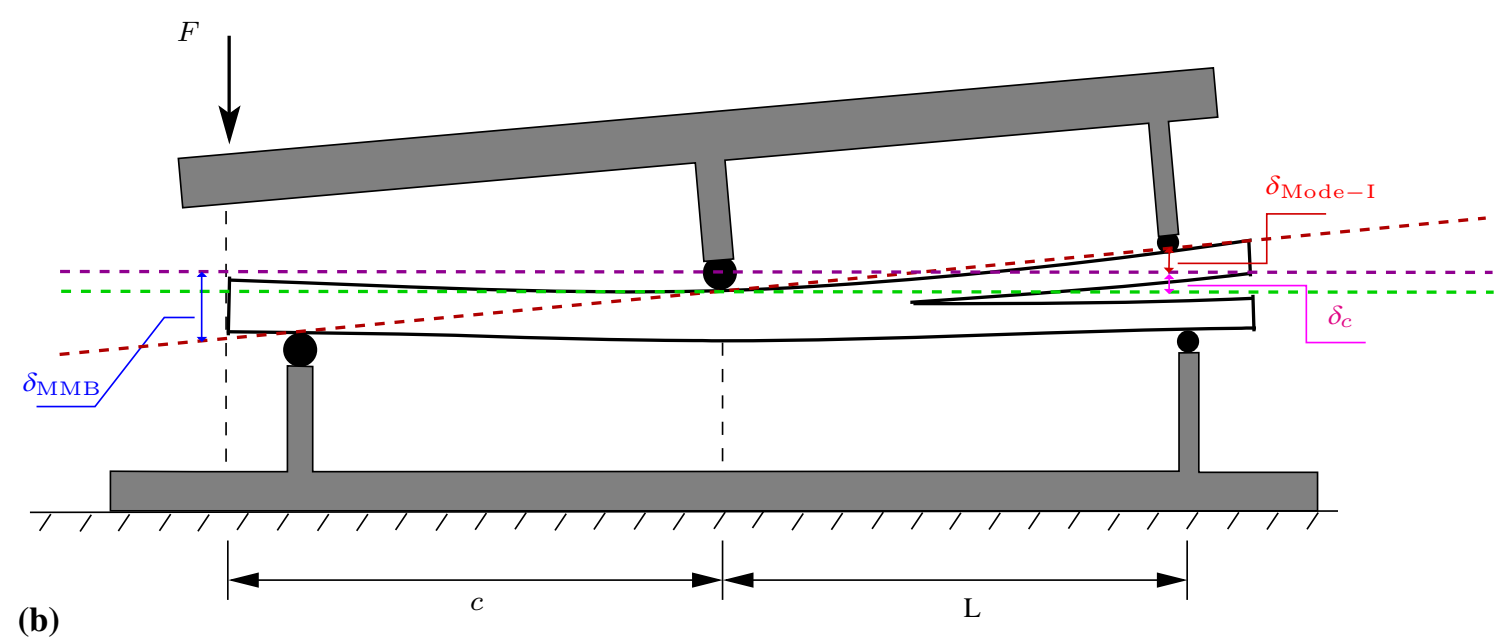

Fig. 9 a Geometrical sketch of the MMB testing device with the specimen in the undeformed configuration; $\mathbf{b}$ in the deformed configuration with the corresponding displacements (reconstructed from Crews and Reeder [12]

increment, where $F_{\text {hinge }}$ is the reaction force at the hinge. In addition, (57) is exploited so as to compute the corresponding values of $\delta_{\mathrm{MMB}}$.

\subsubsection{Results and discussion}

The anisotropic phase-field approach is examined for unidirectional composite laminates by comparing the experimental data with the finite element results for the two different mode mixtures namely $30 \%$ and $50 \%$, see Fig. $12 \mathrm{a}$, where a slight anisotropy is incorporated into the phase-field model $\left(\omega_{\mathrm{f}_{0}}=1\right)$. A close examination indicates an agreement between the experimental and numerical results for the two mode mixtures. The largely linear initial response of the unidirectional laminate precedes with a rather abrupt decline, leading the fracture process zone to grow. The growth shows an inclined pattern towards the top of the specimen while rupturing several laminae on its way, as presented in Fig. 14a.
The simulations performed for $\omega_{\mathrm{f}_{0}}=30$ are primarily in line with those conducted for $\omega_{\mathrm{f}_{0}}=1$ during the initial phase of macro-cracking, giving rise to a sharp reduction in the load bearing capacity, as indicated in Fig. 12b. Nevertheless, the material tends to sustain a constant amount of load as the crack propagates further, while damaging the interlaminar medium between the laminae. The crack path starts to better trail the direction along which the reinforcing fibers are embedded, see Fig. 14b, which stands in sharp contrast to the former, i.e. $\omega_{\mathrm{f}_{0}}=1$.

If we are to assess the discrepancy created when a relatively strong anisotropic geometric resistance to cracking is assumed $\left(\omega_{\mathrm{f}_{0}}=30\right)$, it is evident that the crack no longer cut across the laminae located on the top of each other, but starts to evolve at the interface between the laminae towards the right end of the specimen, thereby resembling peel tests, see, e.g., Gültekin et al. [19], where a relatively constant load drives the peeling of the specimen parallel to the fibers in the 
Fig. 10 Illustration of loading of the MMB test specimen decomposed according to Mode-I and Mode-II cases (reconstructed from Crews and Reeder [12]
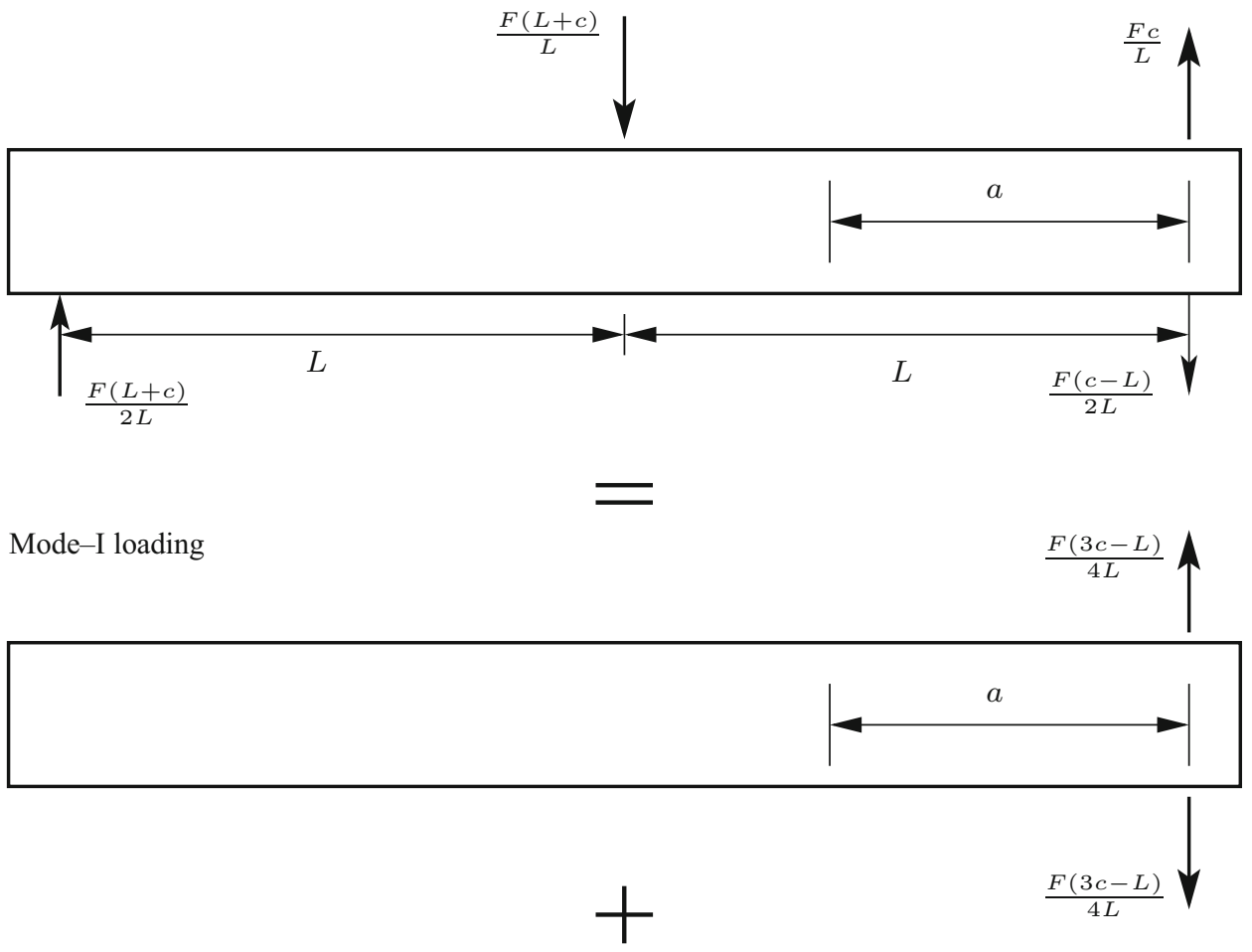

Mode-II loading
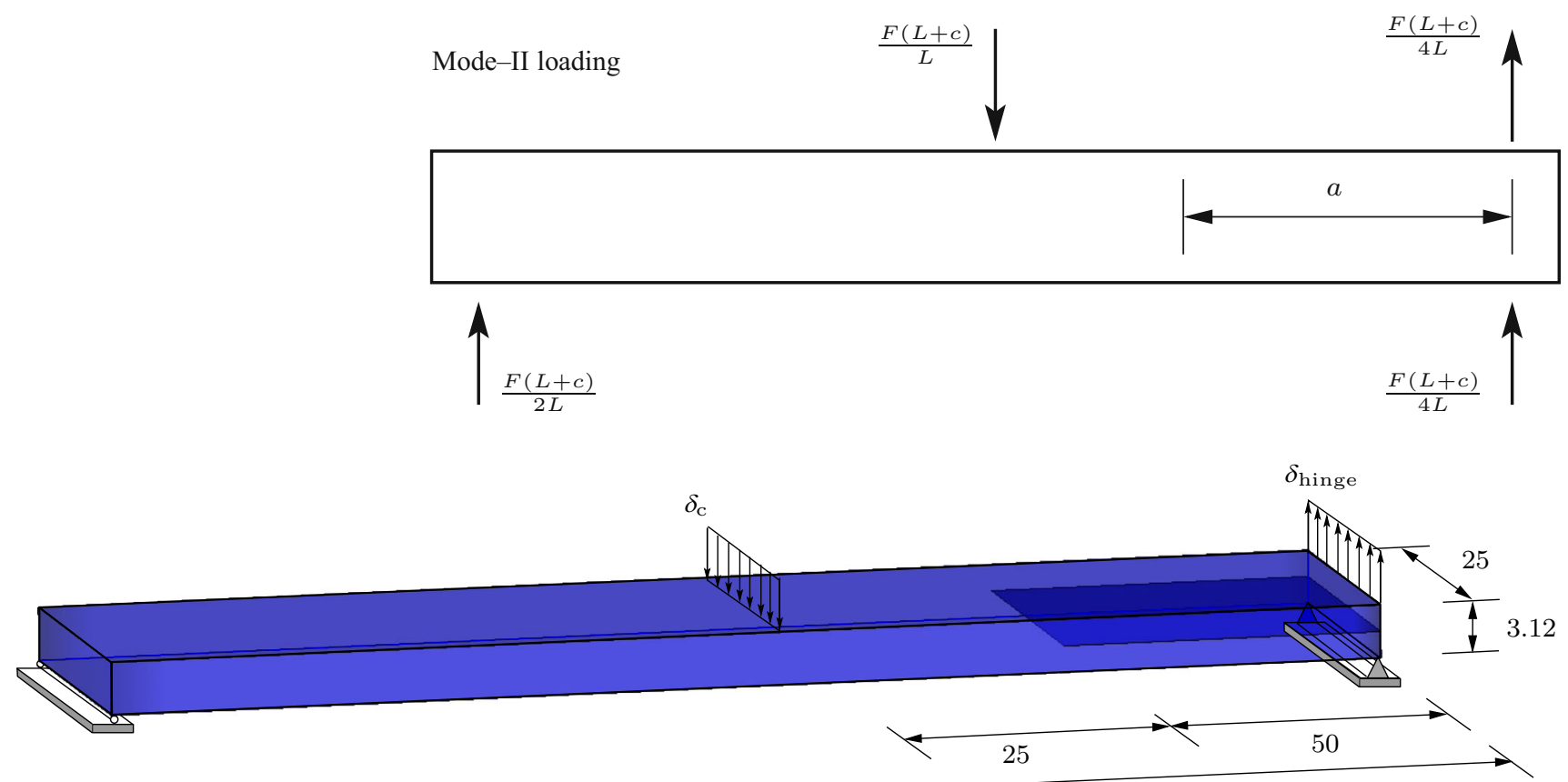

150

Fig. 11 Dimensions of the specimen with a $50 \mathrm{~mm}$ film placed in the mid-plane serving as a delamination initiator. Also shown are the boundary conditions and displacement at the hinge and the middle of the specimen, i.e. $\delta_{\text {hinge }}$ and $\delta_{\mathrm{c}}$. All dimensions are in millimeters 
Fig. 12 Experimental (dashed curves) and numerical (solid curves) results for the relationship between the load $F$ and the loading point displacement $\delta_{\mathrm{MMB}}$ for unidirectional laminates with $30 \%$ and $50 \%$ mode mixtures: a $\omega_{\mathrm{f}_{0}}=1 ; \mathbf{b} \omega_{\mathrm{f}_{0}}=30$
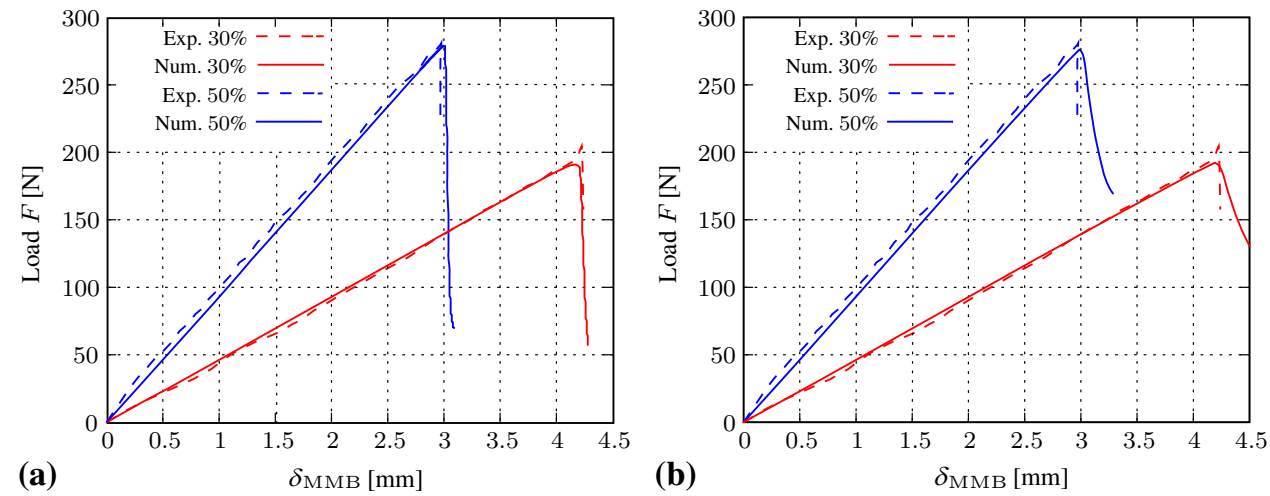

Table 2 Model parameters $\lambda, \mu, \mu_{\mathrm{f}}, g_{\mathrm{c}}^{\text {iso }}$ and $g_{\mathrm{c}}^{\text {ani }}$ of an APC2 lamina with related values and units

\begin{tabular}{lll}
\hline Parameter & Value & Unit \\
\hline$\lambda$ & $5.84 \times 10^{3}$ & $(\mathrm{MPa})$ \\
$\mu$ & $3.61 \times 10^{3}$ & $(\mathrm{MPa})$ \\
$\mu_{\mathrm{f}}$ & $62.0 \times 10^{3}$ & $(\mathrm{MPa})$ \\
$g_{\mathrm{c}}^{\text {iso }}$ & 1.82 & $(\mathrm{MPa} \mathrm{mm})$ \\
$g_{\mathrm{c}}^{\text {ani }}$ & 33.0 & $(\mathrm{MPamm})$ \\
\hline
\end{tabular}

post-cracking phase. However, in the case of $\omega_{\mathrm{f}_{0}}=1$ the crack evolution is driven across the laminate until the top of the specimen, which eventually results in the failure of one of the arms. As a consequence, a more abrupt failure of the entire system occurs, which is seen in Fig. 12a.

\section{Conclusion}

This study presents one of the few attempts to model failure of engineered composite materials by making use of an anisotropic crack phase-field approach. A concise yet critical outline of previous contributions pertaining to strength-based and energy-based criteria for failure of composites is given. Subsequently, the theoretical backbones of the anisotropic phase-field approach is reviewed which relies on the superposition of the distinct fracture processes associated with the matrix and fibrous content. The phase-field model of fracture
Table 3 Lengths $c$, proportional loads and related displacements for each mode mixture

\begin{tabular}{lll}
\hline Mode mixture & $30 \%$ & $50 \%$ \\
\hline$c(\mathrm{~mm})$ & 98.5 & 65.0 \\
Hinge load (N)/displacement $(\mathrm{mm})$ & $1.58 / 2.14$ & $1.04 / 1.34$ \\
Middle load $(\mathrm{N}) /$ displacement $(\mathrm{mm})$ & $2.58 / 1.0$ & $2.04 / 1.0$ \\
\hline
\end{tabular}

is essentially modular consisting of two sub-problems emanating from the deformation field $\varphi$ and the crack phase-field $d$. The numerical examples and the discussions focus on the crack initiation via fitting to experimental data and the direction on which the crack finds its path through relevant tests, such as Mode-I, Mode-II and MMB. In particular, the first example scrutinizes the anisotropic fracture of a single-edged notch plate in response to Mode-I and Mode-II loadings by altering the orientation of fibers, while the second example offers an analysis of a unidirectional CFRP laminate under $\mathrm{MMB}$, touching upon different fracture zones in relation to the anisotropy parameter. It is noteworthy that the fit of the anisotropic model shows a satisfactory coherence with experimental data. The proposed model approach serves a sound basis for more advanced analyses of crack initiation and propagation in unidirectional fiber-reinforced polymer matrix composites.
Fig. 13 Numerical prediction versus experimental data for an APC2 lamina-stress $\sigma$ versus strain $\varepsilon$ in the 1 (horizontal) and 2 (vertical) direction: a tension in the 1 direction; $\mathbf{b}$ compression in the 2 direction
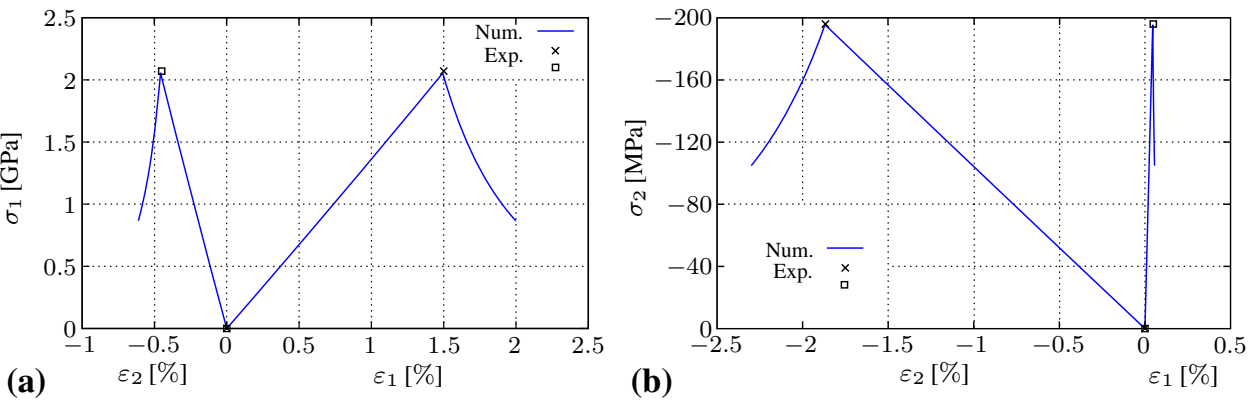
Fig. 14 Evolution of the crack phase-field $d$ with respect to the mode mixture of $30 \%$ (left panels) and 50\% (right panels) for two anisotropy parameters: a $\omega_{\mathrm{f}_{0}}=1 ; \mathbf{b} \omega_{\mathrm{f}_{0}}=30$
$30 \%$ mode mixture

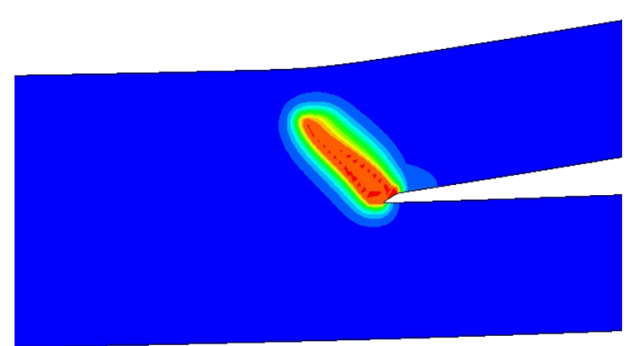

(a)

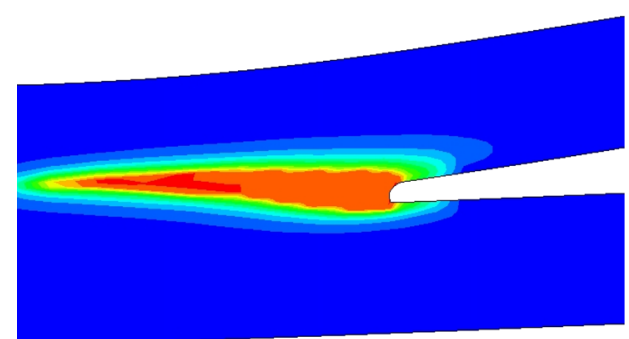

(b)

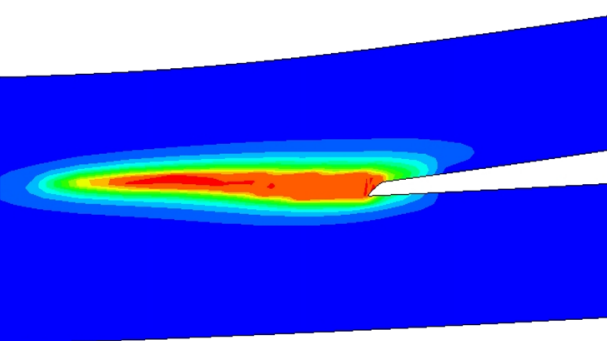

$50 \%$ mode mixture
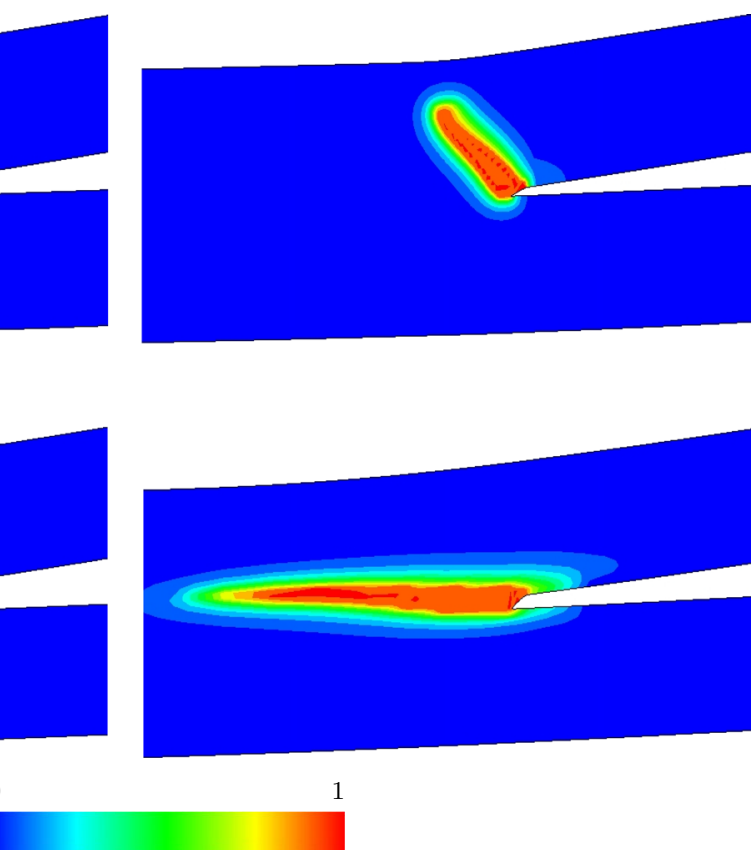

$d$

Acknowledgements H.D. gratefully acknowledges the financial support from Tübitak grant scheme Bideb 2232 under Grant Number $114 \mathrm{C} 073$.

\section{References}

1. ASTM International (2001) ASTM D6671-01 standards test method for mixed mode I-mode II interlaminar fracture toughness of unidirectional fiber reinforced polymer matrix composites. ASTM International, West Conshohocken

2. Alessi R, Freddi F (2017) Phase-field modelling of failure in hybrid laminates. Compos Struct 181:9-25

3. Ambati M, Gerasimov T, De Lorenzis L (2015) Phase-field modeling of ductile fracture. Comput Mech 55:1017-1040

4. Arteiro A, Catalanotti G, Reinoso J, Linde P, Camanho PP (2019) Simulation of the mechanical response of thin-ply composites: from computational micro-mechanics to structural analysis. Arch Comput Methods Eng 26:1445-1487

5. Argon AS (1972) Fracture of composites. Treatise Mater Sci Technol 1:79-114

6. Azzi VD, Tsai SW (1965) Anisotropic strength of composites. Exp Mech 5:283-288

7. Betten J (1987) Formulation of anisotropic constitutive equations. In: Boehler JP (ed) Applications of tensor functions in solid mechanics. Springer, Wien, pp 228-250 CISM Courses and Lectures no. 292

8. Borden MJ, Hughes TJR, Landis CM, Anvari A (2016) A phasefield formulation for fracture in ductile materials: Finite deformation balance law derivation, plastic degradation, and stress triaxiality effects. Comput Methods Appl Mech Eng 312:130-166

9. Boehler JP (1979) A simple derivation of representations for nonpolynomial constitutive equations in some cases of anisotropy. ZAMM Z Angew Math Mech 59:157-167
10. Cahn JW, Hilliard JE (1958) Free energy of a nonuniform system. I. Interfacial free energy. J Chem Phys 28:258-267

11. Clayton JD, Knap J (2015) Phase field modeling of directional fracture in anisotropic polycrystals. Comput Mater Sci 98:158-169

12. Crews JH Jr, Reeder JR (1988) A mixed-mode bending apparatus for delamination testing. NASA TM, 100662. Technical Report

13. Cuntze RG (2006) Efficient 3D and 2D failure conditions for UD laminae and their application within the verification of the laminate design. Compos Sci Technol 66:1081-1096

14. Dal H, Gültekin O, Denli FA, Holzapfel GA (2017) Phase-field models for the failure of anisotropic continua. Proc Appl Math Mech 17:91-94

15. Francfort GA, Marigo J-J (1998) Revisiting brittle fracture as an energy minimization problem. J Mech Phys Solids 46:1319-1342

16. Griffith AA (1921) The phenomena of rupture and flow in solids. Philos Trans R Soc A 221:163-197

17. Grogan DM, Leen SB, Brádaigh CMÓ (2014) An XFEM-based methodology for fatigue delamination and permeability of composites. Compos Struct 107:205-218

18. Gültekin O, Dal H, Holzapfel GA (2016) A phase-field approach to model fracture of arterial walls: Theory and finite element analysis. Comput Methods Appl Mech Eng 312:542-566

19. Gültekin O, Dal H, Holzapfel GA (2018) Numerical aspects of failure in soft biological tissues favor energy-based criteria: a ratedependent anisotropic crack phase-field model. Comput Methods Appl Mech Eng 331:23-52

20. Hashin Z (1980) Failure criteria for unidirectional fiber composites. J Appl Mech 47:329-334

21. Hashin Z (1996) Finite thermoelastic fracture criterion with application to laminate cracking analysis. J Mech Phys Solids 44:11291145

22. Hill R (1998) The mathematical theory of plasticity. Oxford University Press, New York

23. Holzapfel GA (2000) Nonlinear solid mechanics. A continuum approach for engineering. Wiley, Chichester 
24. Holzapfel GA, Gasser TC, Ogden RW (2000) A new constitutive framework for arterial wall mechanics and a comparative study of material models. J Elast 61:1-48

25. Li B, Peco C, Millán D, Arias I, Arroyo M (2015) Phase-field modeling and simulation of fracture in brittle materials with strongly anisotropic surface energy. Int J Numer Methods Eng 102:711-727

26. Marsden JE, Hughes TJR (1994) Mathematical foundations of elasticity. Dover, New York

27. Miehe C, Welschinger F, Hofacker M (2010) Thermodynamically consistent phase-field models of fracture: variational principles and multi-field FE implementations. Int J Numer Methods Eng 83:1273-1311

28. Miehe C, Hofacker M, Welschinger F (2010) A phase field model for rate-independent crack propagation: robust algorithmic implementation based on operator splits. Comput Methods Appl Mech Eng 199:2765-2778

29. Miehe C, Schänzel LM, Ulmer H (2015) Phase field modeling of fracture in multi-physics problems. Part I. Balance of crack surface and failure criteria for brittle crack propagation in thermo-elastic solid. Comput Methods Appl Mech Eng 294:449-485

30. Miehe C, Hofacker M, Schänzel LM, Aldakheel F (2015) Phase field modeling of fracture in multi-physics problems. Part II. Coupled brittle-to-ductile failure criteria and crack propagation in thermo-elastic-plastic solids. Comput Methods Appl Mech Eng 294:486-522

31. Miehe C, Dal H, Schänzel LM, Raina A (2016) A phase-field model for chemo-mechanical induced fracture in lithium-ion battery electrode particles. Int J Numer Methods Eng 106:683-711

32. Nairn JA (1989) The strain-energy release rate of composite microcracking-a variational approach. J Compos Mater 23:11061129

33. Naghipour P, Bartsh M, Voggenreiter H (2011) Simulation and experimental validation of mixed mode delamination in multidirectional CF/PEEK laminates under fatigue loading. Int J Solids Struct 48:1070-1081

34. Naghipour P (2011) Numerical simulations and experimental investigations on quasi-static and cyclic mixed mode delamination of multidirectional CFRP laminates. Ph.D. Thesis, University of Stuttgart

35. Naghipour P, Schneider J, Bartsch M, Hausmann J, Voggenreiter H (2009) Fracture simulation of CFRP laminates in mixed mode bending. Eng Fract Mech 76:2821-2833

36. Nguyen T-T, Réthéro J, Yvonnet J, Baietto M-C (2017) Multiphase-field modeling of anisotropic crack propagation for polycrystalline materials. Comput Mech 60:289-314

37. Puck A, Schürmann H (1998) Failure analysis of FRP laminates by means of physically based phenomenological models. Compos Sci Technol 58:1045-1067

38. Qiu G, Pence T (1997) Remarks on the behavior of simple directionally reinforced incompressible nonlinearly elastic solids. J Elast 49:1-30
39. Reinoso J, Arteiro A, Paggi M, Camanho PP (2017) Strength prediction of notched thin ply laminates using finite fracture mechanics and the phase field approach. Compos Sci Technol 58:205-216

40. Schreiber C, Kuhn C, Müller R (2018) Phase field modeling of brittle fracture in materials with anisotropic fracture resistance. PAMM 18:e201800113

41. Schröder J, Neff P (2003) Invariant formulation of hyperelastic transverse isotropy based on polyconvex free energy functions. Int J Solids Struct 40:401-445

42. Soden PD, Hinton MJ, Kaddour AS (2004) Lamina properties, lay-up configurations and loading conditions for a range of fibre reinforced composite laminates. In: Hinton M, Soden PD, Kaddour A-S (eds) Failure criteria in fibre-reinforced polymer composites. Elsevier, Amsterdam, pp 30-51

43. Spencer A (1972) Deformations of fibre-reinforced materials. Oxford University Press, Oxford

44. Teichtmeister S, Kienle D, Aldakheel F, Keip M-A (2017) Phasefield modeling of fracture in anisotropic brittle solids. Int J NonLinear Mech 97:1-21

45. Talreja R, Singh CV (2012) Damage and failure of composite materials. Cambridge University Press, New York

46. Talreja R (2014) Assessment of the fundamentals of failure theories for composite materials. Compos Sci Technol 105:190-201

47. Tsai SW, Wu EM (1971) General theory of strength for anisotropic materials. J Compos Mater 5:58-80

48. Turon A, Dávila CG, Camanho PP, Costa J (2007) An engineering solution for mesh size effects in the simulation of delamination using cohesive zone models. Eng Fract Mech 74:1665-1682

49. von Mises R (1913) Mechanik der festen Körper im plastisch deformablen Zustand. Göttin Nachr Math Phys 1:582-592

50. Wang Y, Waisman H (2015) Progressive delamination analysis of composite materials using XFEM and a discrete damage zone mode. Comput Mech 55:1-26

51. Wolfe WE, Butalia TS (1998) A strain-energy based failure criterion for non-linear analysis of composite laminates subjected to biaxial loading. Compos Sci Technol 58:1107-1124

52. Yang Q, Cox B (2005) Cohesive models for damage evolution in laminated composites. Int J Fract 133:107-137

53. Yazdani S, Rust WJH, Wriggers P (2016) An XFEM approach for modelling delamination in composite laminates. Compos Struct 135:353-364

54. Zhao L, Gong Y, Zhang J, Chen Y, Fei B (2016) Simulation of delamination growth in multidirectional laminates under mode I and mixed mode I/II loadings using cohesive elements. Compos Struct 116:509-522

Publisher's Note Springer Nature remains neutral with regard to jurisdictional claims in published maps and institutional affiliations. 